\title{
Nitrile-Promoted Rh-Catalyzed Intermolecular Hydroacylation of Olefins with Salicylaldehyde
}

Masanori Imai, ${ }^{\mathrm{a}}$ Masakazu Tanaka, ${ }^{\mathrm{b} *}$ Shinji Nagumo, ${ }^{\mathrm{a} \dagger}$ Norio Kawahara, ${ }^{\mathrm{a}}$ and Hiroshi Suemune ${ }^{\mathrm{b} *}$

${ }^{a}$ Hokkaido College of Pharmacy, Hokkaido 047-0264, Japan

${ }^{\mathrm{b}}$ Graduate School of Pharmaceutical Sciences, Kyushu University, Fukuoka 812-8582, Japan

mtanaka@phar.kyushu-u.ac.jp; suemune@phar.kyushu-u.ac.jp

* To whom correspondence should be addressed.

Tel: + 81-92-642-6604. Fax: + 81-92-642-6545.

Supporting Information

Synthetic Scheme of Substrates 8 and 9

Copies of ${ }^{1} \mathrm{H}$ NMR and ${ }^{13} \mathrm{C}$ NMR spectra 
Synthetic Scheme of Substrates 8 and $\mathbf{9}$

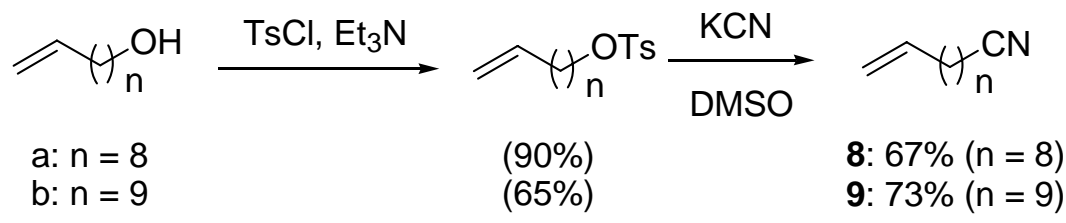




\section{Experimental Section}

General. ${ }^{1} \mathrm{H}$ NMR spectra were determined at $270 \mathrm{MHz}$ or $400 \mathrm{MHz}$ unless otherwise noted. Infrared spectra were recorded on a JASCO FT/IR-7000 spectrometer. EIMS spectra were taken on a Hitachi M-2000 mass spectrometer. The melting points were measured with Yanagimoto micro-melting point apparatus and are uncorrected.

Undec-10-enenitrile (8). A mixture of dec-9-en-1-ol (1.70 g, $10.9 \mathrm{mmol}), \mathrm{Et}_{3} \mathrm{~N}(2.30 \mathrm{~mL}$, $16.4 \mathrm{mmol})$, and p-toluenesulfonyl chloride $(2.12 \mathrm{~g}, 10.9 \mathrm{mmol})$ in $\mathrm{CH}_{2} \mathrm{Cl}_{2}(20 \mathrm{~mL})$ was stirred overnight at room temperature. Then, the solution was diluted with water, extracted with $\mathrm{CHCl}_{3}$, washed with saturated aqueous $\mathrm{NH}_{4} \mathrm{Cl}$, and dried over $\mathrm{MgSO}_{4}$. After removal of the solvent, the residue was purified by column chromatography on silica gel (30\% EtOAc in hexane) to give tosylate $(3.05 \mathrm{~g}, 90 \%)$ as a colorless oil: IR (neat) 2928, 2860, 1640, $1601 \mathrm{~cm}^{-1} ;{ }^{1} \mathrm{H} \mathrm{NMR}\left(\mathrm{CDCl}_{3}\right)$ $\delta 7.79$ (br d, $J=8.8 \mathrm{~Hz}, 2 \mathrm{H}), 7.34$ (br d, $J=8.8 \mathrm{~Hz}, 2 \mathrm{H}), 5.80(\mathrm{~m}, 1 \mathrm{H}), 4.92-5.00(\mathrm{~m}, 2 \mathrm{H}), 4.02(\mathrm{t}$, $J=6.8 \mathrm{~Hz}, 2 \mathrm{H}$ ), 2.45 (s, 3H), 2.02 (quartet, $J=6.8 \mathrm{~Hz}, 2 \mathrm{H}$ ), 1.63 (quintet, $J=6.8 \mathrm{~Hz}, 2 \mathrm{H}$ ), 1.23 $1.36(\mathrm{~m}, 10 \mathrm{H})$. A mixture of the tosylate $(3.05 \mathrm{~g}, 9.82 \mathrm{mmol})$ and $\mathrm{KCN}(730 \mathrm{mg}, 10.8 \mathrm{mmol})$ in DMSO $(30 \mathrm{~mL})$ was stirred overnight at room temperature. Then, the solution was diluted with water, extracted with ether, washed with $5 \%$ aqueous $\mathrm{NaHCO}_{3}$, and dried over $\mathrm{MgSO}_{4}$. After removal of the solvent, the residue was purified by column chromatography on silica gel $(20 \%$ EtOAc in hexane) to give $8(1.09 \mathrm{~g}, 67 \%)$ as a colorless oil: IR (neat) 2928, 2860, 2250, $1642 \mathrm{~cm}^{-}$ ${ }^{1} ;{ }^{1} \mathrm{H}$ NMR $\left(\mathrm{CDCl}_{3}\right) \delta 5.76-5.86(\mathrm{~m}, 1 \mathrm{H}), 4.92-5.02(\mathrm{~m}, 2 \mathrm{H}), 2.33(\mathrm{t}, J=7.3 \mathrm{~Hz}, 2 \mathrm{H}), 2.04$ (quartet, $J=7.3 \mathrm{~Hz}, 2 \mathrm{H}$ ), 1.65 (quintet, $J=7.3 \mathrm{~Hz}, 2 \mathrm{H}$ ), $1.31-1.46$ (m, 10H); EI-HRMS calcd for $\mathrm{C}_{11} \mathrm{H}_{19} \mathrm{~N}\left(\mathrm{M}^{+}\right)$165.1517, found $165.1500 .^{\mathrm{S} 1}$

Dodec-11-enenitrile (9). Compound 9 was prepared from undec-10-en-1-ol in a manner similar to that describe for the preparation of 8. A colorless oil: IR (neat) 2930, 2860, 2250, $1640 \mathrm{~cm}^{-1} ;{ }^{1} \mathrm{H} \mathrm{NMR}\left(\mathrm{CDCl}_{3}\right) \delta 5.81(\mathrm{~m}, 1 \mathrm{H}), 4.92-5.01(\mathrm{~m}, 2 \mathrm{H}), 2.33(\mathrm{t}, J=6.8 \mathrm{~Hz}, 2 \mathrm{H}), 2.04$ (quartet, $J=6.8 \mathrm{~Hz}, 2 \mathrm{H}$ ), 1.66 (quintet, $J=6.8 \mathrm{~Hz}, 2 \mathrm{H}$ ), $1.29-1.46$ (m, 12H); EI-HRMS calcd for $\mathrm{C}_{12} \mathrm{H}_{21} \mathrm{~N}\left(\mathrm{M}^{+}\right)$179.1674, found 179.1690. ${ }^{\mathrm{S} 1}$ 
RhCl( $\left(\mathrm{PPh}_{3}\right)_{3}$-Catalyzed Intermolecular Hydroacylation between Salicylaldehyde (1) and Allyl Cyanide (4). $\quad \mathrm{RhCl}\left(\mathrm{PPh}_{3}\right)_{3}$-catalyzed hydroacylation between salicylaldehyde $\mathbf{1}$ and allyl cyanide 4 at room temperature afforded 10a and 10b in $98 \%$ yield as a mixture of isomers in the ratio of 5 to 1 . Each compound 10a and 10b could be separated by preparative TLC. 5-(2Hydroxyphenyl)-5-oxopentanenitrile (10a). Colorless crystals: $\mathrm{mp} 49.0-50.0{ }^{\circ} \mathrm{C}$ (recryst. from $\mathrm{CDCl}_{3}$-hexane); IR (KBr) 3438 (br), 2958, 2908, 2250, 1644, $1613 \mathrm{~cm}^{-1} ;{ }^{1} \mathrm{H} \mathrm{NMR}\left(\mathrm{CDCl}_{3}\right) \delta$ 12.07 (s, 1H), 7.76 (br d, $J=8 \mathrm{~Hz}, 1 \mathrm{H}), 7.49$ (br t, $J=8 \mathrm{~Hz}, 1 \mathrm{H}), 7.00$ (br d, $J=8 \mathrm{~Hz}, 1 \mathrm{H}), 6.92$ (br t, $J=8 \mathrm{~Hz}, 1 \mathrm{H}), 3.22(\mathrm{t}, J=7.0 \mathrm{~Hz}, 2 \mathrm{H}), 2.54(\mathrm{t}, J=7.0 \mathrm{~Hz}, 2 \mathrm{H}), 2.13$ (quintet, $J=7.0 \mathrm{~Hz}, 2 \mathrm{H}$ ); EIHRMS calcd for $\mathrm{C}_{11} \mathrm{H}_{11} \mathrm{NO}_{2}\left(\mathrm{M}^{+}\right)$189.0790, found 189.0812. Anal. Calcd for $\mathrm{C}_{11} \mathrm{H}_{11} \mathrm{NO}_{2}$ : C, 69.83; H, 5.86; N, 7.40; Found: C, 69.96; H, 5.97; N, 7.26. 4-(2-Hydroxyphenyl)-3-methyl-4oxobutyronitrile (10b). A colorless oil: IR (neat) 3024, 2982, 2252, 1640, $1615 \mathrm{~cm}^{-1}$; ${ }^{1} \mathrm{H}$ $\operatorname{NMR}\left(\mathrm{CDCl}_{3}\right) \delta 12.00(\mathrm{~s}, 1 \mathrm{H}), 7.71($ br d, $J=8 \mathrm{~Hz}, 1 \mathrm{H}), 7.52$ (br t, $\left.J=8 \mathrm{~Hz}, 1 \mathrm{H}\right), 7.03$ (br d, $J=8$ Hz, 1H), 6.95 (br t, $J=8 \mathrm{~Hz}, 1 \mathrm{H}$ ), 3.86 (sestet, $J=7.3 \mathrm{~Hz}, 1 \mathrm{H}$ ), 2.75 (dd, $J=6.9,16.9 \mathrm{~Hz}, 1 \mathrm{H}$ ), $2.64(\mathrm{dd}, J=7.7,16.9 \mathrm{~Hz}, 1 \mathrm{H}), 1.47(\mathrm{~d}, J=6.9 \mathrm{~Hz}, 3 \mathrm{H})$; EI-HRMS calcd for $\mathrm{C}_{11} \mathrm{H}_{11} \mathrm{NO}_{2}$ $\left(\mathrm{M}^{+}\right) 189.0790$, found 189.0760 .

\section{RhCl( $\left(\mathrm{PPh}_{3}\right)_{3}$-Catalyzed Intermolecular Hydroacylation between Salicylaldehyde (1)} and 4-Pentenenitrile (5). $\quad \mathrm{RhCl}\left(\mathrm{PPh}_{3}\right)_{3}$-catalyzed hydroacylation between salicylaldehyde 1 and 4-pentenenitrile 5 at room temperature afforded 11 in 94\% yield as a mixture of isomers in the ratio of $>20(n)$ to 1 (i). Colorless crystals: $\mathrm{mp} 83.0-84.0{ }^{\circ} \mathrm{C}$ (recryst. from $\mathrm{CDCl}_{3}$-hexane); IR (KBr) 3448 (br), 2946, 2878, 2242, 1642, $1615 \mathrm{~cm}^{-1}$; ${ }^{1} \mathrm{H}$ NMR $\left(\mathrm{CDCl}_{3}\right) \delta 12.23$ (s, 1H), 7.74 (br d, $J=8 \mathrm{~Hz}, 1 \mathrm{H}), 7.47$ (br t, $J=8 \mathrm{~Hz}, 1 \mathrm{H}), 6.98$ (br d, $J=8 \mathrm{~Hz}, 1 \mathrm{H}), 6.91$ (br t, $J=8 \mathrm{~Hz}, 1 \mathrm{H}), 3.07$ (t, $J=6.8 \mathrm{~Hz}, 2 \mathrm{H}), 2.42(\mathrm{t}, J=6.8 \mathrm{~Hz}, 2 \mathrm{H}$ ), 1.91 (quintet, $J=6.8 \mathrm{~Hz}, 2 \mathrm{H}$ ), 1.77 (quintet, $J=6.8 \mathrm{~Hz}$, $2 \mathrm{H}) ;{ }^{13} \mathrm{C} \mathrm{NMR}\left(100 \mathrm{MHz}, \mathrm{CDCl}_{3}\right) \delta 205.2,162.4,136.4,129.7,119.3,119.1,118.9,118.5,37.0$, 24.9, 23.0, 17.1; EI-HRMS calcd for $\mathrm{C}_{12} \mathrm{H}_{13} \mathrm{NO}_{2}\left(\mathrm{M}^{+}\right)$203.0946, found 203.0935. Anal. Calcd for $\mathrm{C}_{12} \mathrm{H}_{13} \mathrm{NO}_{2}$ : C, 70.92; H, 6.45; N, 6.89; Found: C, 70.99; H, 6.57; N, 6.77.

\section{RhCl $\left(\mathrm{PPh}_{3}\right)_{3}$-Catalyzed Intermolecular Hydroacylation between Salicylaldehyde (1) and}

5-Hexenenitrile (6). $\quad \mathrm{RhCl}\left(\mathrm{PPh}_{3}\right)_{3}$-catalyzed hydroacylation between salicylaldehyde 1 and 5hexenenitrile 6 at room temperature afforded 12 in $83 \%$ yield as a mixture of isomers in the ratio of 
$>20(n)$ to $1(i) . \quad$ Colorless crystals: $\quad \mathrm{mp} 74.0-75.0{ }^{\circ} \mathrm{C}$ (recryst. from $\mathrm{CDCl}_{3}$-hexane); IR $(\mathrm{KBr})$ 3452 (br), 2950, 2878, 2248, 1644, $1615 \mathrm{~cm}^{-1} ;{ }^{1} \mathrm{H} \mathrm{NMR}\left(\mathrm{CDCl}_{3}\right) \delta 12.29$ (s, 1H), 7.75 (br d, $J=8$ $\mathrm{Hz}, 1 \mathrm{H}), 7.47$ (br t, $J=8 \mathrm{~Hz}, 1 \mathrm{H}), 6.99$ (br d, $J=8 \mathrm{~Hz}, 1 \mathrm{H}), 6.90$ (br t, $J=8 \mathrm{~Hz}, 1 \mathrm{H}), 3.03$ (t, $J=$ $6.8 \mathrm{~Hz}, 2 \mathrm{H}), 2.38(\mathrm{t}, J=6.8 \mathrm{~Hz}, 2 \mathrm{H}), 1.70-1.83(\mathrm{~m}, 4 \mathrm{H}), 1.53-1.60(\mathrm{~m}, 2 \mathrm{H}) ;{ }^{13} \mathrm{C}$ NMR $(100$ $\left.\mathrm{MHz}, \mathrm{CDCl}_{3}\right) \delta 205.9,162.2,136.1,129.7,119.4,119.0,118.8,118.3,37.5,28.0,25.0,23.0,16.8$; EI-HRMS calcd for $\mathrm{C}_{13} \mathrm{H}_{15} \mathrm{NO}_{2}\left(\mathrm{M}^{+}\right)$217.1103, found 217.1077. Anal. Calcd for $\mathrm{C}_{13} \mathrm{H}_{15} \mathrm{NO}_{2}$ : C, 71.87; H, 6.96; N, 6.45; Found: C, 71.81; H, 7.06; N, 6.34.

\section{RhCl(PPh $)_{3}$-Catalyzed Intermolecular Hydroacylation between Salicylaldehyde (1) and} 6-Heptenenitrile (7). $\quad \mathrm{RhCl}\left(\mathrm{PPh}_{3}\right)_{3}$-Catalyzed hydroacylation between salicylaldehyde 1 and 6heptenenitrile $\mathbf{7}$ at room temperature afforded $\mathbf{1 3}$ in 99\% yield as a mixture of isomers in the ratio of $>20(n)$ to $1(i) . \quad$ Colorless crystals: $\mathrm{mp} 60.0-61.0{ }^{\circ} \mathrm{C}$ (recryst. from $\mathrm{CDCl}_{3}$-hexane); $\mathrm{IR}(\mathrm{KBr})$ 3452 (br), 2944, 2868, 2252, 1636, $1618 \mathrm{~cm}^{-1}$; ${ }^{1} \mathrm{H}$ NMR $\left(\mathrm{CDCl}_{3}\right) \delta 12.29$ (s, 1H), 7.76 (br d, $J=8$ $\mathrm{Hz}, 1 \mathrm{H}), 7.47$ (br t, $J=8 \mathrm{~Hz}, 1 \mathrm{H}), 6.98$ (br d, $J=8 \mathrm{~Hz}, 1 \mathrm{H}), 6.90$ (br t, $J=8 \mathrm{~Hz}, 1 \mathrm{H}), 3.01$ (t, $J=$ $7.3 \mathrm{~Hz}, 2 \mathrm{H}$ ), 2.36 (t, $J=7.3 \mathrm{~Hz}, 2 \mathrm{H}$ ), 1.78 (quintet, $J=7.3 \mathrm{~Hz}, 2 \mathrm{H}$ ), 1.69 (quintet, $J=7.3 \mathrm{~Hz}, 2 \mathrm{H}$ ), $1.42-1.56(\mathrm{~m}, 4 \mathrm{H}) ;{ }^{13} \mathrm{C} \mathrm{NMR}\left(100 \mathrm{MHz}, \mathrm{CDCl}_{3}\right) \delta 206.3,162.3,136.2,129.8,119.6,119.1,118.8$, 118.4, 37.8, 28.3, 28.2, 25.0, 23.8, 16.9; EI-HRMS calcd for $\mathrm{C}_{14} \mathrm{H}_{17} \mathrm{NO}_{2}\left(\mathrm{M}^{+}\right)$231.1259, found 231.1245. Anal. Calcd for $\mathrm{C}_{14} \mathrm{H}_{17} \mathrm{NO}_{2}$ : C, 72.70; H, 7.41; N, 6.06; Found: C, 72.82; H, 7.54; N, 5.96.

\section{$\mathrm{RhCl}\left(\mathrm{PPh}_{3}\right)_{3}$-Catalyzed Intermolecular Hydroacylation between Salicylaldehyde (11)}

and Undec-10-enenitrile (8). $\quad \mathrm{RhCl}\left(\mathrm{PPh}_{3}\right)_{3}$-catalyzed hydroacylation between salicylaldehyde 1 and undec-10-enenitrile $\mathbf{8}$ at room temperature afforded $\mathbf{1 4}$ in 70\% yield as a mixture of isomers in the ratio of $>20(n)$ to 1 (i). Colorless crystals: mp $74.0-75.0{ }^{\circ} \mathrm{C}$ (recryst. from $\mathrm{CDCl}_{3}$ hexane); IR (KBr) 3428 (br), 2922, 2854, 2244, 1638, $1611 \mathrm{~cm}^{-1} ;{ }^{1} \mathrm{H}$ NMR $\left(\mathrm{CDCl}_{3}\right) \delta 12.40$ (s, 1H), 7.76 (br d, $J=8 \mathrm{~Hz}, 1 \mathrm{H}), 7.45$ (br t, $J=8 \mathrm{~Hz}, 1 \mathrm{H}$ ), 6.97 (br d, $J=8 \mathrm{~Hz}, 1 \mathrm{H}), 6.89$ (br t, $J=8$ $\mathrm{Hz}, 1 \mathrm{H}), 2.98$ (t, $J=7.3 \mathrm{~Hz}, 2 \mathrm{H}), 2.32$ (t, $J=7.3 \mathrm{~Hz}, 2 \mathrm{H}$ ), 1.73 (quintet, $J=7.3 \mathrm{~Hz}, 2 \mathrm{H}$ ), 1.64 (quintet, $J=7.3 \mathrm{~Hz}, 2 \mathrm{H}), 1.30-1.45(\mathrm{~m}, 12 \mathrm{H}) ;{ }^{13} \mathrm{C} \mathrm{NMR}\left(100 \mathrm{MHz}, \mathrm{CDCl}_{3}\right) \delta 206.9,162.5,136.2$, $130.0,119.8,119.3,118.8,118.5,38.3,29.3,29.24,29.20,29.18,28.7,28.6,25.3,24.4$, 17.1; EI- 
HRMS calcd for $\mathrm{C}_{18} \mathrm{H}_{25} \mathrm{NO}_{2}\left(\mathrm{M}^{+}\right)$287.1885, found 287.1903. Anal. Calcd for $\mathrm{C}_{18} \mathrm{H}_{25} \mathrm{NO}_{2}$ : C, 75.22; H, 8.77; N, 4.87; Found: C, 75.40; H, 8.67; N, 4.86.

\section{RhCl(PPh $)_{3}$-Catalyzed Intermolecular Hydroacylation between Salicylaldehyde (1) and}

Dodec-11-enenitrile (9). $\quad \mathrm{RhCl}\left(\mathrm{PPh}_{3}\right)_{3}$-catalyzed hydroacylation between salicylaldehyde 1 and dodec-11-enenitrile $\mathbf{9}$ at room temperature afforded $\mathbf{1 5}$ in 80\% yield as a mixture of isomers in the ratio of $>20(n)$ to 1 (i). Colorless crystals: $\operatorname{mp~} 73.0-74.0{ }^{\circ} \mathrm{C}$ (recryst. from $\mathrm{CHCl}_{3}$-hexane); IR (KBr) 3484 (br), 2924, 2854, 2248, 1640, $1618 \mathrm{~cm}^{-1} ;{ }^{1} \mathrm{H}$ NMR $\left(\mathrm{CDCl}_{3}\right) \delta 12.39$ (s, 1H), 7.76 (br d, $J=8 \mathrm{~Hz}, 1 \mathrm{H}), 7.46$ (br t, $J=8 \mathrm{~Hz}, 1 \mathrm{H}), 6.98$ (br d, $J=8 \mathrm{~Hz}, 1 \mathrm{H}), 6.89$ (br t, $J=8 \mathrm{~Hz}, 1 \mathrm{H}$ ), $2.98(\mathrm{t}, J=7.3 \mathrm{~Hz}, 2 \mathrm{H}), 2.33(\mathrm{t}, J=7.3 \mathrm{~Hz}, 2 \mathrm{H}$ ), 1.74 (quintet, $J=7.3 \mathrm{~Hz}, 2 \mathrm{H}$ ), 1.65 (quintet, $J=$ $7.3 \mathrm{~Hz}, 2 \mathrm{H}), 1.29-1.46(\mathrm{~m}, 14 \mathrm{H}) ;{ }^{13} \mathrm{C} \mathrm{NMR}\left(100 \mathrm{MHz}, \mathrm{CDCl}_{3}\right) \delta 206.8,162.3,136.0,129.9$, $119.7,119.2,118.7,118.3,38.1,29.22,29.20$ (x2), 29.1 (x2), 28.6, 28.5, 25.2, 24.3, 16.9; EIHRMS calcd for $\mathrm{C}_{19} \mathrm{H}_{27} \mathrm{NO}_{2}\left(\mathrm{M}^{+}\right)$301.2042, found 301.2043. Anal. Calcd for $\mathrm{C}_{19} \mathrm{H}_{27} \mathrm{NO}_{2}$ : $\mathrm{C}$, 75.71; H, 9.03; N, 4.65; Found: C, 75.88; H, 9.10; N, 4.59.

$\mathrm{RhCl}\left(\mathrm{PPh}_{3}\right)_{3}$-Catalyzed Intermolecular Hydroacylation between Salicylaldehyde (1) and 2-Methyl-1,5-hexadiene (16). ${ }^{3, \mathrm{a}, \mathrm{b}} \quad \mathrm{RhCl}\left(\mathrm{PPh}_{3}\right)_{3}$-catalyzed hydroacylation between salicylaldehyde 1 and 2-methyl-1,5-hexadiene $\mathbf{1 6}$ at room temperature afforded 25a and 25b in $95 \%$ yield as a mixture of isomers in the ratio of 1 (normal) to 10 (iso). A colorless oil: IR

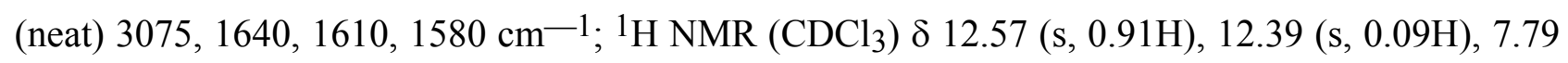
(br d, $J=8 \mathrm{~Hz}, 1 \mathrm{H}), 7.47$ (br t, $J=8 \mathrm{~Hz}, 1 \mathrm{H}), 6.99$ (br d, $J=8 \mathrm{~Hz}, 1 \mathrm{H}), 6.90$ (br t, $J=8 \mathrm{~Hz}, 1 \mathrm{H}$ ), 4.72 (br s, 1H), 4.65 (br s, 1H), $3.53(\mathrm{~m}, 0.91 \mathrm{H}), 3.00(\mathrm{t}, J=7.3 \mathrm{~Hz}, 0.18 \mathrm{H}), 1.95-2.20(\mathrm{~m}, 3.09 \mathrm{H})$, $1.71(\mathrm{~s}, 3 \mathrm{H}), 1.55-1.75(\mathrm{~m}, 1.18 \mathrm{H}), 1.24(\mathrm{~d}, J=6.9 \mathrm{~Hz}, 2.73 \mathrm{H})$.

\section{$\mathrm{RhCl}\left(\mathrm{PPh}_{3}\right)_{3}$-Catalyzed Intermolecular Hydroacylation between Salicylaldehyde (1) and}

1,5-heptadiene (17). ${ }^{\text {a,b }} \quad \mathrm{RhCl}\left(\mathrm{PPh}_{3}\right)_{3}$-catalyzed hydroacylation between salicylaldehyde $\mathbf{1}$ and 1,5-heptadiene $\mathbf{1 7}$ at room temperature afforded $\mathbf{2 6 a}, \mathbf{b}, \mathbf{c}$ in $82 \%$ yield as a mixture of isomers in the

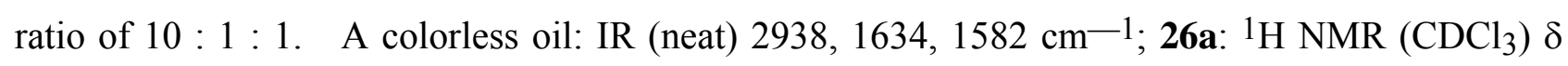
12.39 (s, 1H), 7.76 (br d, $J=8 \mathrm{~Hz}, 1 \mathrm{H}), 7.46$ (br t, $J=8 \mathrm{~Hz}, 1 \mathrm{H}), 6.98$ (br d, $J=8 \mathrm{~Hz}, 1 \mathrm{H}), 6.89$ (br 
$\mathrm{t}, J=8 \mathrm{~Hz}, 1 \mathrm{H}), 5.30-5.55(\mathrm{~m}, 2 \mathrm{H}), 2.95-3.02(\mathrm{~m}, 2 \mathrm{H}), 2.00-2.15(\mathrm{~m}, 2 \mathrm{H}), 1.15-1.80(\mathrm{~m}, 7 \mathrm{H})$. Each compound 26a,b,c could be separated by preparative TLC. ${ }^{3 a, b}$

RhCl(PPh $)_{3}$-Catalyzed Intermolecular Hydroacylation between Salicylaldehyde (1) and 1,7-Octadiene (18). $\quad \mathrm{RhCl}\left(\mathrm{PPh}_{3}\right)_{3}$-catalyzed hydroacylation between salicylaldehyde 1 and 1,7octadiene $\mathbf{1 8}$ at room temperature afforded 27 in $73 \%$ yield as a mixture of isomers in the ratio of $>20(n)$ to 1 (i). A colorless oil; IR (neat) 3080, 2932, 1642, $1615 \mathrm{~cm}^{-1} ;{ }^{1} \mathrm{H}$ NMR $\left(\mathrm{CDCl}_{3}\right) \delta$ 12.39 (s, 1H), 7.76 (br d, $J=8.1 \mathrm{~Hz}, 1 \mathrm{H}), 7.45$ (br t, $J=8.3 \mathrm{~Hz}, 1 \mathrm{H}), 6.98$ (br d, $J=8.3 \mathrm{~Hz}, 1 \mathrm{H}$ ), $6.89($ br t $, J=8.1 \mathrm{~Hz}, 1 \mathrm{H}), 5.81(\mathrm{~m}, 1 \mathrm{H}), 4.92-5.02(\mathrm{~m}, 2 \mathrm{H}), 2.98(\mathrm{t}, J=7.3 \mathrm{~Hz}, 2 \mathrm{H}), 2.05(\mathrm{~m}, 2 \mathrm{H})$, 1.75 (quintet, $J=6.8 \mathrm{~Hz}, 2 \mathrm{H}), 1.35-1.44(\mathrm{~m}, 6 \mathrm{H}) ;{ }^{13} \mathrm{C} \mathrm{NMR}\left(100 \mathrm{MHz}, \mathrm{CDCl}_{3}\right) \delta 206.7,162.4$, 138.8, 136.0, 129.9, 119.2, 118.7, 118.4, 114.2, 38.1, 33.6, 29.0, 28.8, 28.6, 24.3; EI-HRMS calcd for $\mathrm{C}_{15} \mathrm{H}_{20} \mathrm{O}_{2}\left(\mathrm{M}^{+}\right)$232.1463, found 232.1466.

RhCl(PPh$)_{3}$-Catalyzed Intermolecular Hydroacylation between Salicylaldehyde (1) and 2,2-Diallylmalonic Acid Diethyl Ester (19). $\quad \mathrm{RhCl}\left(\mathrm{PPh}_{3}\right)_{3}$-catalyzed hydroacylation between salicylaldehyde $\mathbf{1}$ and 2,2-diallylmalonic acid diethyl ester $\mathbf{1 9}$ at room temperature afforded $\mathbf{2 8}$ in $80 \%$ yield as a colorless oil.; IR (neat) $3082,2984,1734,1642,1615 \mathrm{~cm}^{-1} ;{ }^{1} \mathrm{H}_{\mathrm{NMR}}\left(\mathrm{CDCl}_{3}\right) \delta$ 12.30 (s, 1H), 7.72 (br d, $J=8 \mathrm{~Hz}, 1 \mathrm{H}), 7.46$ (br t, $J=8 \mathrm{~Hz}, 1 \mathrm{H}), 6.98$ (br d, $J=8 \mathrm{~Hz}, 1 \mathrm{H}), 6.89$ (br t, $J=8 \mathrm{~Hz}, 1 \mathrm{H}), 5.67(\mathrm{~m}, 1 \mathrm{H}), 5.09-5.14(\mathrm{~m}, 2 \mathrm{H}), 4.19(\mathrm{q}, J=7.1 \mathrm{~Hz}, 4 \mathrm{H}), 3.01(\mathrm{t}, J=7.1 \mathrm{~Hz}$, 2H), $2.70(\mathrm{~d}, J=7.6 \mathrm{~Hz}, 2 \mathrm{H}), 1.93-1.98(\mathrm{~m}, 2 \mathrm{H}), 1.61-1.72(\mathrm{~m}, 2 \mathrm{H}), 1.25(\mathrm{t}, J=7.1 \mathrm{~Hz}, 6 \mathrm{H})$;

${ }^{13} \mathrm{C}$ NMR $\left(100 \mathrm{MHz}, \mathrm{CDCl}_{3}\right) \delta 205.5,170.9$ (x2), 162.3, 136.1, 132.2, 129.7, 119.1, 118.9, 118.7, 118.4, 61.1 (x2), 57.2, 38.0, 36.8, 31.6, 18.5, 14.0 (x2); EI-HRMS calcd for $\mathrm{C}_{20} \mathrm{H}_{26} \mathrm{O}_{6}\left(\mathrm{M}^{+}\right)$ 362.1729 , found 362.1729 .

\section{$\mathrm{RhCl}\left(\mathrm{PPh}_{3}\right)_{3}$-Catalyzed Intermolecular Hydroacylation between Salicylaldehyde (1) and} 1-Hexene (20). ${ }^{3 a, b} \quad \mathrm{RhCl}\left(\mathrm{PPh}_{3}\right)_{3}$-catalyzed hydroacylation between salicylaldehyde $\mathbf{1}$ and 1hexene 20 at room temperature afforded 29 in 86\% yield (ratio: $>20: 1$ ) as a colorless oil: IR (neat)

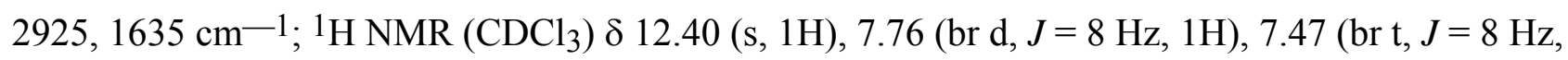
1H), 6.99 (br d, $J=8 \mathrm{~Hz}, 1 \mathrm{H}), 6.89$ (br t, $J=8 \mathrm{~Hz}, 1 \mathrm{H}), 2.99$ (t, $J=7.3 \mathrm{~Hz}, 2 \mathrm{H}), 1.75$ (m, 2H), $1.25-1.50(\mathrm{~m}, 6 \mathrm{H}), 0.90(\mathrm{t}, J=6.9 \mathrm{~Hz}, 3 \mathrm{H})$. 
$\operatorname{RhCl}\left(\mathrm{PPh}_{3}\right)_{3}$-Catalyzed Intermolecular Hydroacylation between Salicylaldehyde (1) and 3,3-Dimethyl-1-butene (22). $\quad \mathrm{RhCl}\left(\mathrm{PPh}_{3}\right)_{3}$-catalyzed hydroacylation between salicylaldehyde 1 and 3,3-dimethyl-1-butene 22 at room temperature afforded 31 in $82 \%$ yield as a colorless oil.; IR (neat) 2960, 2870, 1642, $1615 \mathrm{~cm}^{-1} ;{ }^{1} \mathrm{H} \mathrm{NMR}\left(\mathrm{CDCl}_{3}\right) \delta 12.39$ (s, 1H), 7.77 (br d, $\left.J=8 \mathrm{~Hz}, 1 \mathrm{H}\right)$, 7.46 (br t, $J=8 \mathrm{~Hz}, 1 \mathrm{H}), 6.98$ (br d, $J=8 \mathrm{~Hz}, 1 \mathrm{H}), 6.90$ (br t, $J=8 \mathrm{~Hz}, 1 \mathrm{H}), 2.93-2.97$ (m, 2H), $1.63-1.67(\mathrm{~m}, 2 \mathrm{H}), 0.97(\mathrm{~s}, 9 \mathrm{H}) ;{ }^{13} \mathrm{C} \mathrm{NMR}\left(68 \mathrm{MHz}, \mathrm{CDCl}_{3}\right) \delta 207.5,162.5,136.1,129.9,119.2$, 118.8, 118.5, 38.3, 34.1, 30.2, 29.1 (x3); EI-HRMS calcd for $\mathrm{C}_{13} \mathrm{H}_{18} \mathrm{O}_{2}\left(\mathrm{M}^{+}\right)$206.1307, found 206.1299.

RhCl(PPh$)_{3}$-Catalyzed Intermolecular Hydroacylation between Salicylaldehyde (1) and N-Allyl-4-methylbenzenesulfonamide (23). $\quad \mathrm{RhCl}\left(\mathrm{PPh}_{3}\right)_{3}$-catalyzed hydroacylation between salicylaldehyde $\mathbf{1}$ and $\mathrm{N}$-allyl-4-methylbenzenesulfonamide 23 at room temperature afforded 32 in $53 \%$ yield as colorless crystals: mp $137.0-138.0{ }^{\circ} \mathrm{C}$ (recryst. from $\mathrm{CHCl}_{3}$-hexane); $\mathrm{IR}(\mathrm{KBr})$ 3336, 2976, 2922, 1638, $1611 \mathrm{~cm}^{-1} ;{ }^{1} \mathrm{H}$ NMR $\left(\mathrm{CDCl}_{3}\right) \delta 12.12(\mathrm{~s}, 1 \mathrm{H}), 7.73(\mathrm{br} \mathrm{d}, J=8 \mathrm{~Hz}, 2 \mathrm{H})$, 7.69 (br d, $J=8 \mathrm{~Hz}, 1 \mathrm{H}$ ), 7.47 (br t, $J=8 \mathrm{~Hz}, 1 \mathrm{H}$ ), 7.28 (br d, $J=8 \mathrm{~Hz}, 2 \mathrm{H}$ ), 6.98 (br d, $J=8 \mathrm{~Hz}$, 1H), 6.89 (br t, $J=8 \mathrm{~Hz}, 1 \mathrm{H}), 4.50$ (t, $J=6.3 \mathrm{~Hz}, 1 \mathrm{H}), 3.05-3.10$ (m, 4H), 2.40 (s, 3H), 1.93 (quintet, $J=6.8 \mathrm{~Hz}, 2 \mathrm{H}$ ); EI-HRMS calcd for $\mathrm{C}_{17} \mathrm{H}_{19} \mathrm{NO}_{4} \mathrm{~S}\left(\mathrm{M}^{+}\right)$333.1035, found 333.1013. Anal. Calcd for $\mathrm{C}_{17} \mathrm{H}_{19} \mathrm{NO}_{4} \mathrm{~S}$ : C, 61.24; H, 5.74; N, 4.20; Found: C, 61.28; H, 5.79; N, 4.14.

RhCl($\left(\mathrm{PPh}_{3}\right)_{3}$-Catalyzed Intermolecular Hydroacylation between Salicylaldehyde (1) and 1-Hexyne (24). $\quad \mathrm{RhCl}\left(\mathrm{PPh}_{3}\right)_{3}$-catalyzed hydroacylation between salicylaldehyde 1 and 1-hexyne 24 at room temperature afforded 33a and 33b in 91\% yield as a mixture of isomers in the ratio of 1.1 to 1. 1-2-(Hydroxyphenyl)hept-2-en-1-one (33a). A colorless oil: IR (neat) 3022, 2962, 1651, 1622, $1591 \mathrm{~cm}^{-1} ;{ }^{1} \mathrm{H}$ NMR $\left(\mathrm{CDCl}_{3}\right) \delta 12.73$ (s, 1H), 7.81 (br d, J=8 Hz, 1H), 7.47 (br t, $J=8 \mathrm{~Hz}, 1 \mathrm{H}), 7.21(\mathrm{dt}, J=15.1,7.1 \mathrm{~Hz}, 1 \mathrm{H}), 6.98-7.05(\mathrm{~m}, 2 \mathrm{H}), 6.90(\mathrm{br} \mathrm{t}, J=8 \mathrm{~Hz}, 1 \mathrm{H}), 2.36$ (m, 2H), 1.53 (quintet, $J=7.3 \mathrm{~Hz}, 2 \mathrm{H}$ ), 1.44 (sestet, $J=7.3 \mathrm{~Hz}, 2 \mathrm{H}), 0.94$ (t, $J=7.3 \mathrm{~Hz}, 3 \mathrm{H}$ ); EIHRMS calcd for $\mathrm{C}_{13} \mathrm{H}_{16} \mathrm{O}_{2} \quad\left(\mathrm{M}^{+}\right)$204.1150, found 204.1163. 2-Butyl-1-(2hydroxyphenyl)propenone (33b). A colorless oil: IR (neat) 3026, 2962, 1624, $1603 \mathrm{~cm}^{-1}$; ${ }^{1} \mathrm{H}$ $\operatorname{NMR}\left(\mathrm{CDCl}_{3}\right) \delta 11.99$ (s, 1H), 7.75 (br d, $\left.J=8 \mathrm{~Hz}, 1 \mathrm{H}\right), 7.48$ (br t, $\left.J=8 \mathrm{~Hz}, 1 \mathrm{H}\right), 7.01$ (br d, $J=8$ 
$\mathrm{Hz}, 1 \mathrm{H}), 6.87$ (br t, $J=8 \mathrm{~Hz}, 1 \mathrm{H}), 5.65(\mathrm{br} \mathrm{s}, 1 \mathrm{H}), 5.38(\mathrm{~s}, 1 \mathrm{H}), 2.47$ (t, $J=7.3 \mathrm{~Hz}, 2 \mathrm{H}), 1.44-1.49$

$(\mathrm{m}, 2 \mathrm{H}), 1.38$ (quintet, $J=7.3 \mathrm{~Hz}, 2 \mathrm{H}), 0.91(\mathrm{t}, J=7.3 \mathrm{~Hz}, 3 \mathrm{H})$; EI-HRMS calcd for $\mathrm{C}_{13} \mathrm{H}_{16} \mathrm{O}_{2}\left(\mathrm{M}^{+}\right)$ 204.1150, found 204.1173.

S1 Camps, F. Eta 1., Synth. Comm. 1988, 18, 445. 


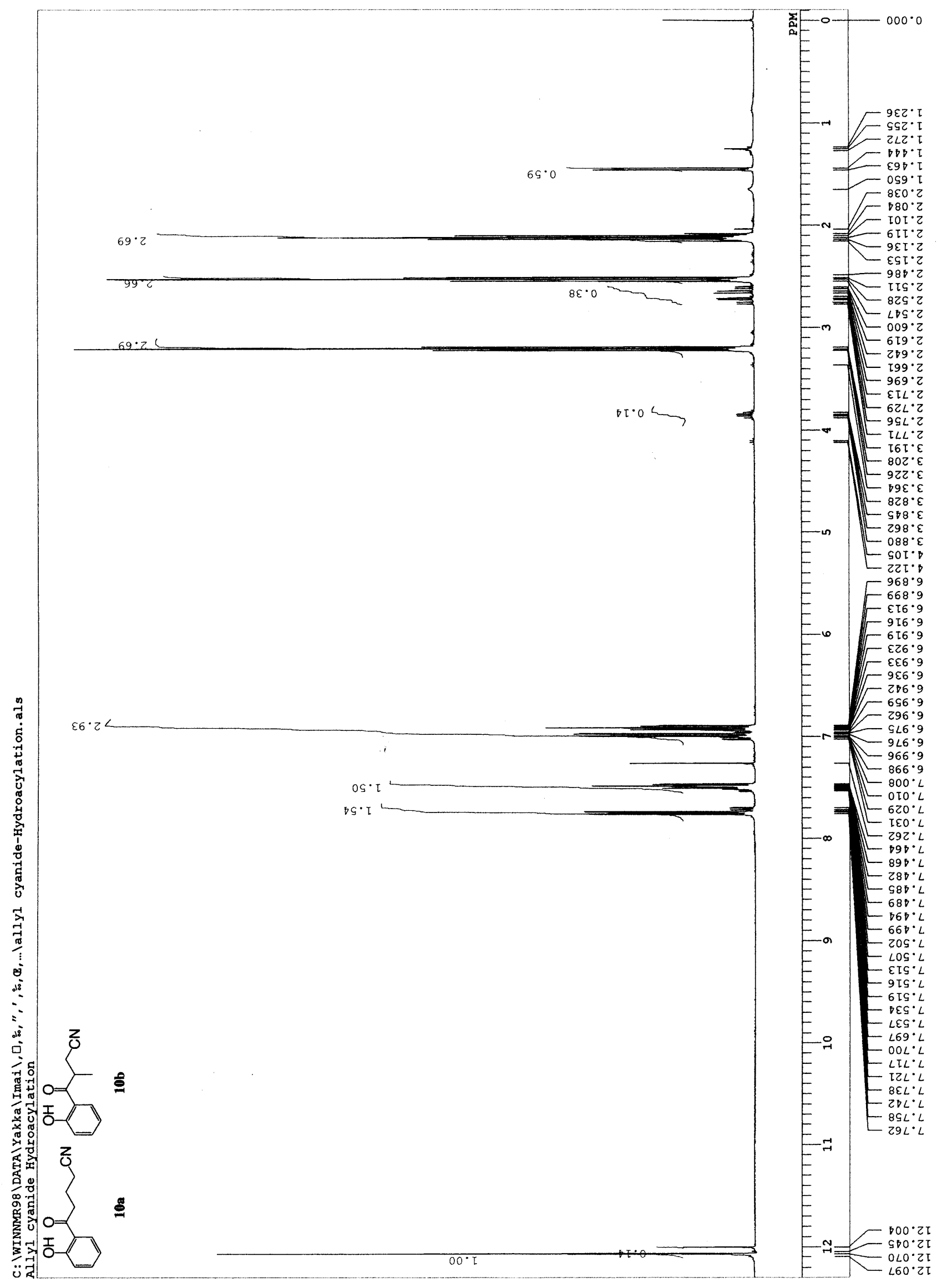




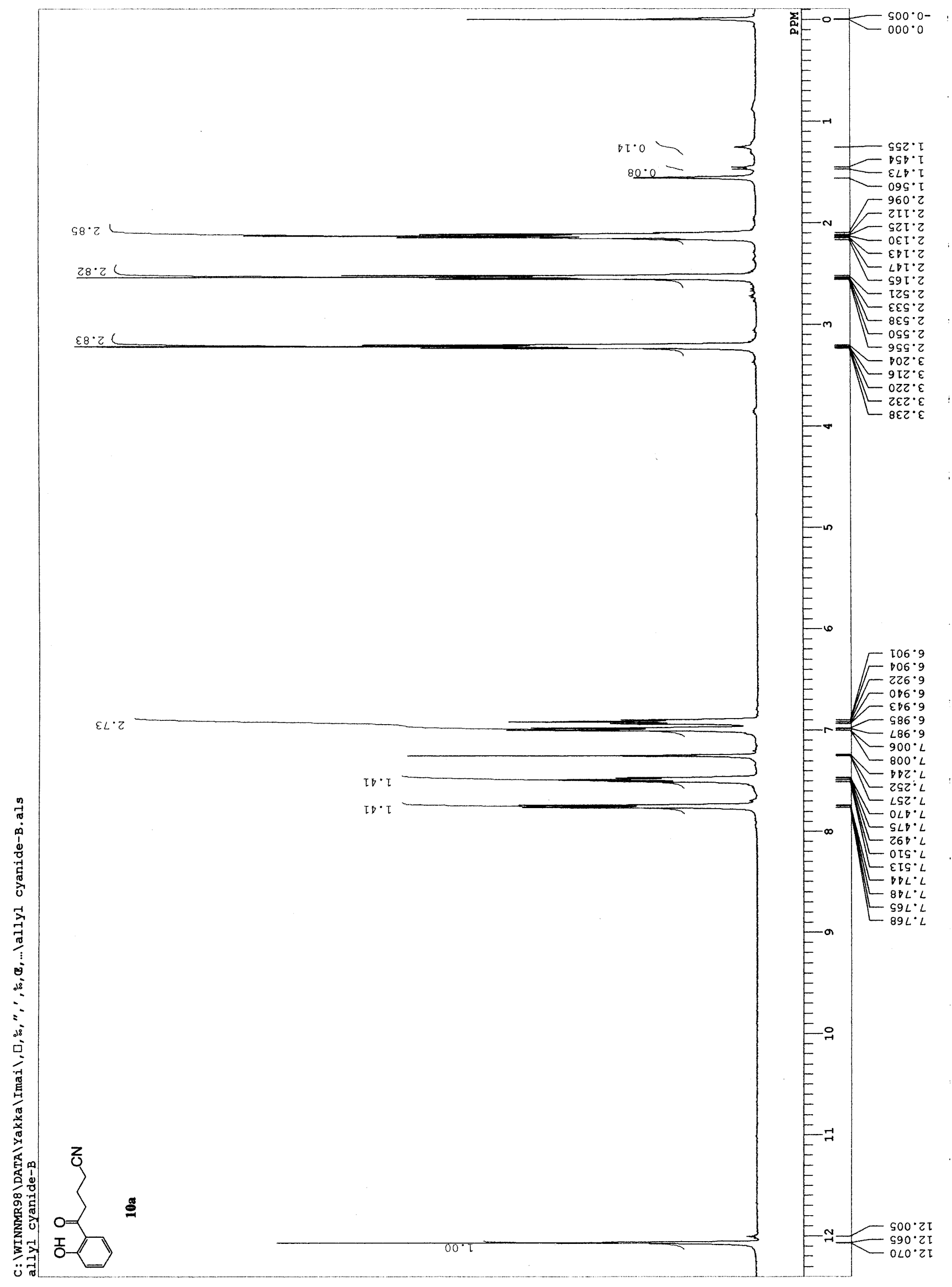




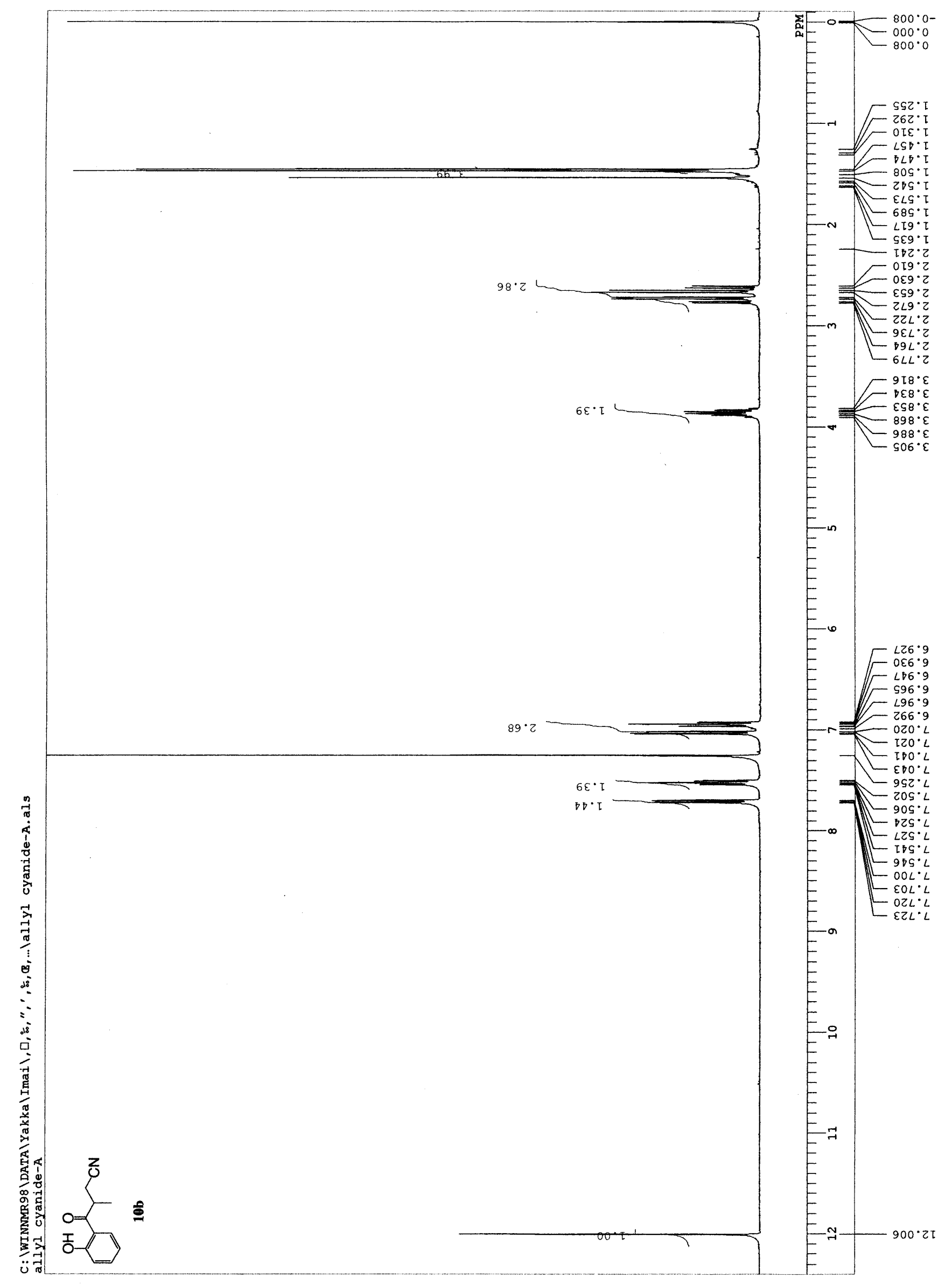




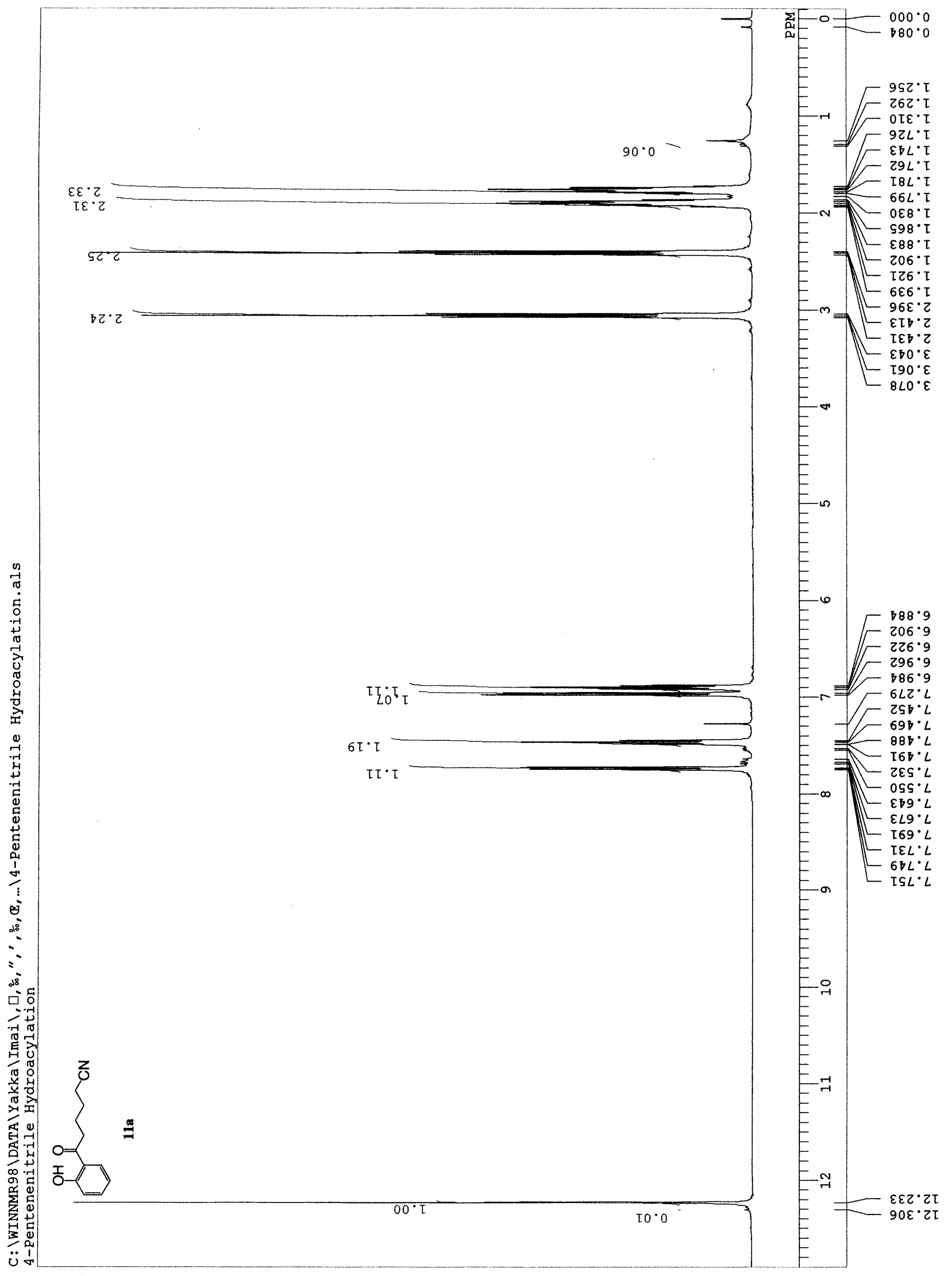




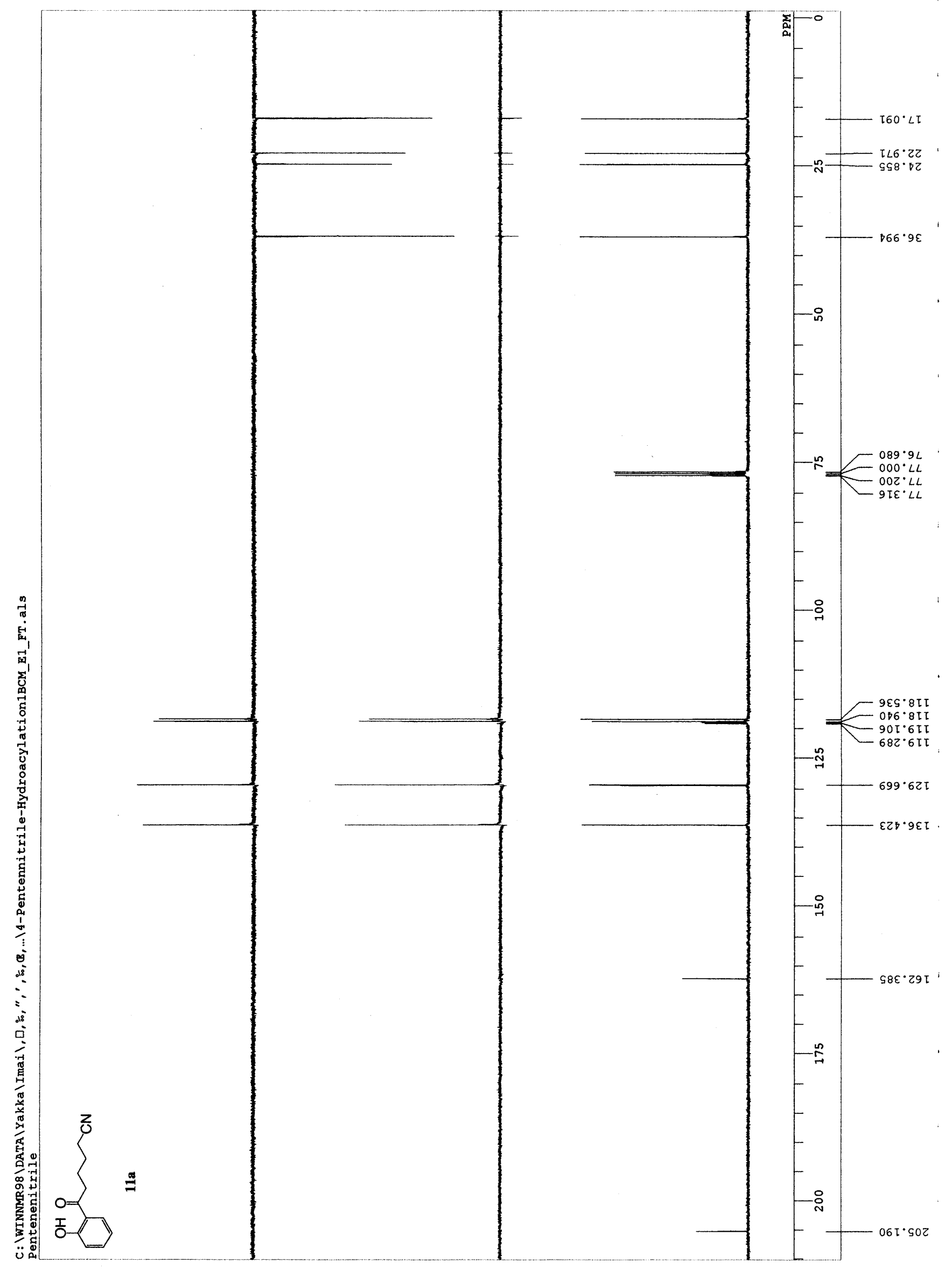




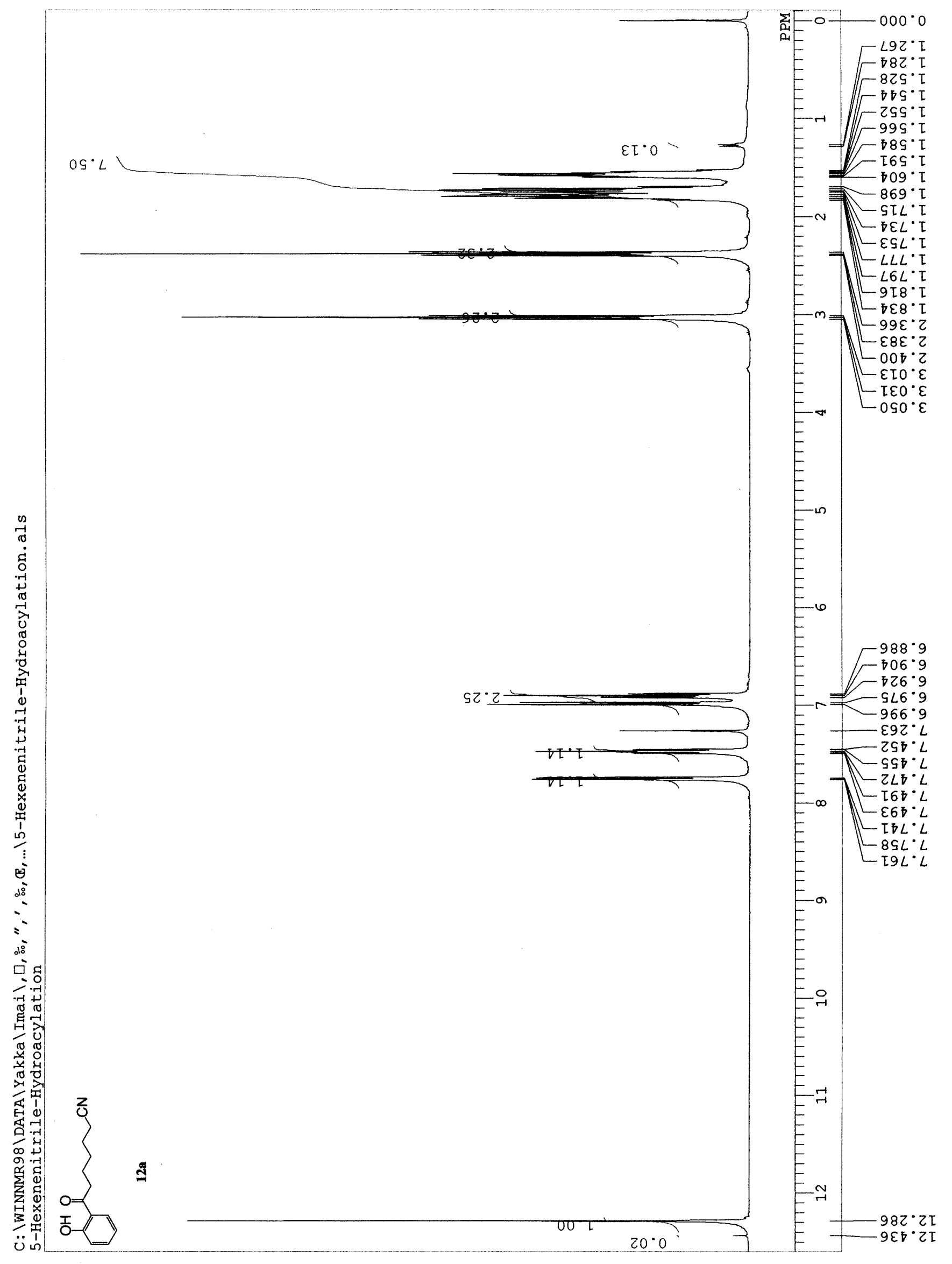




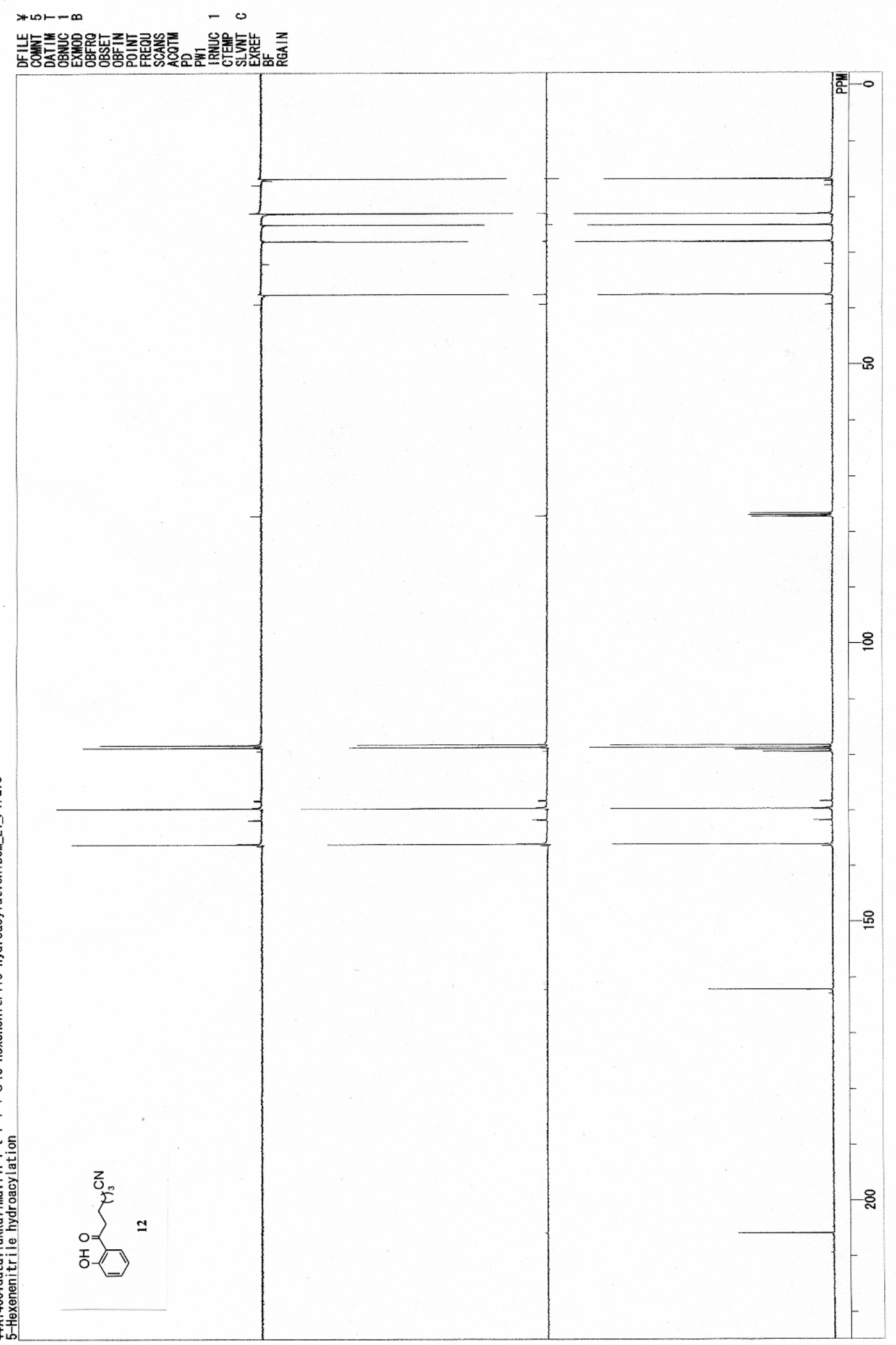




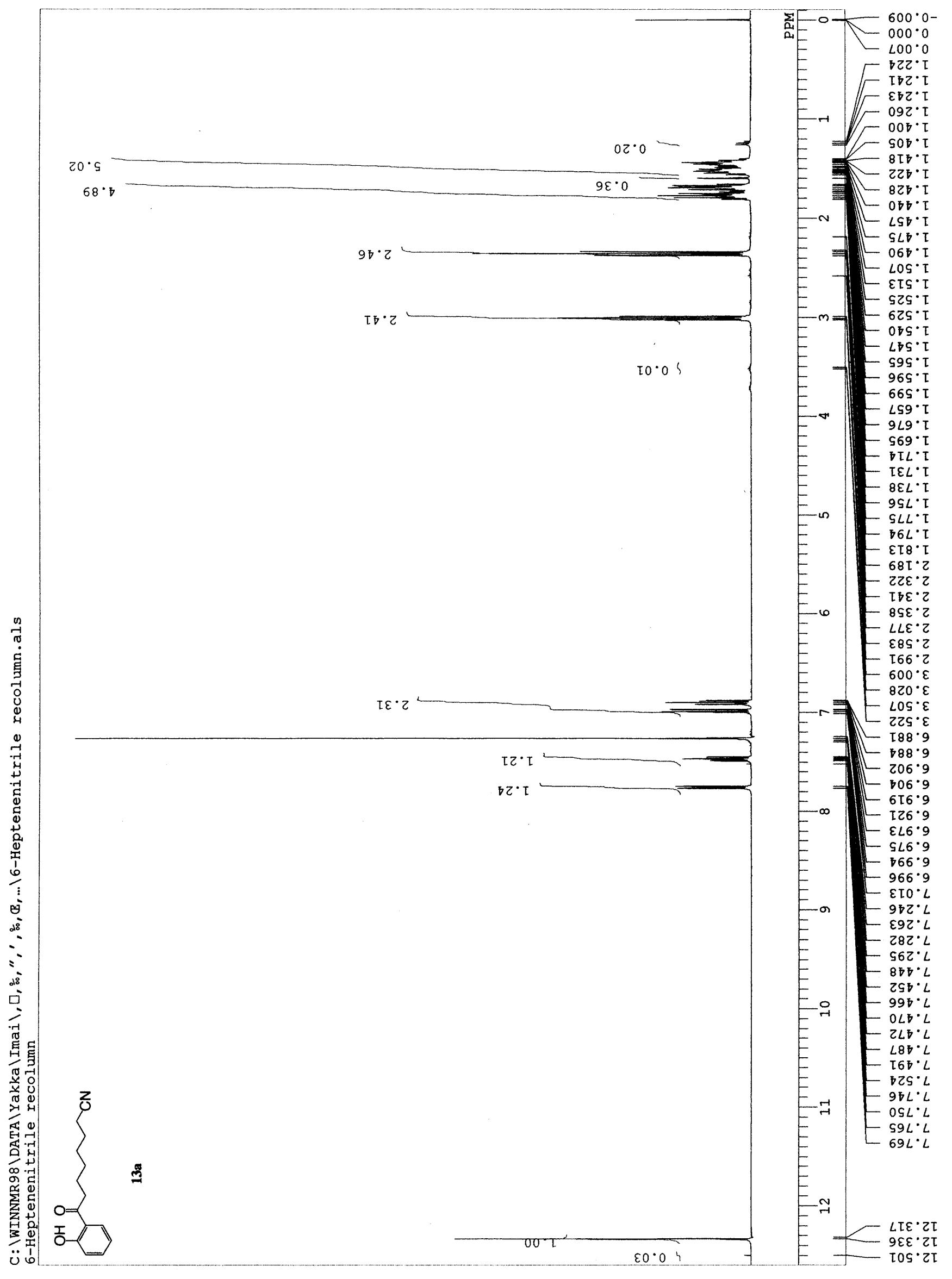




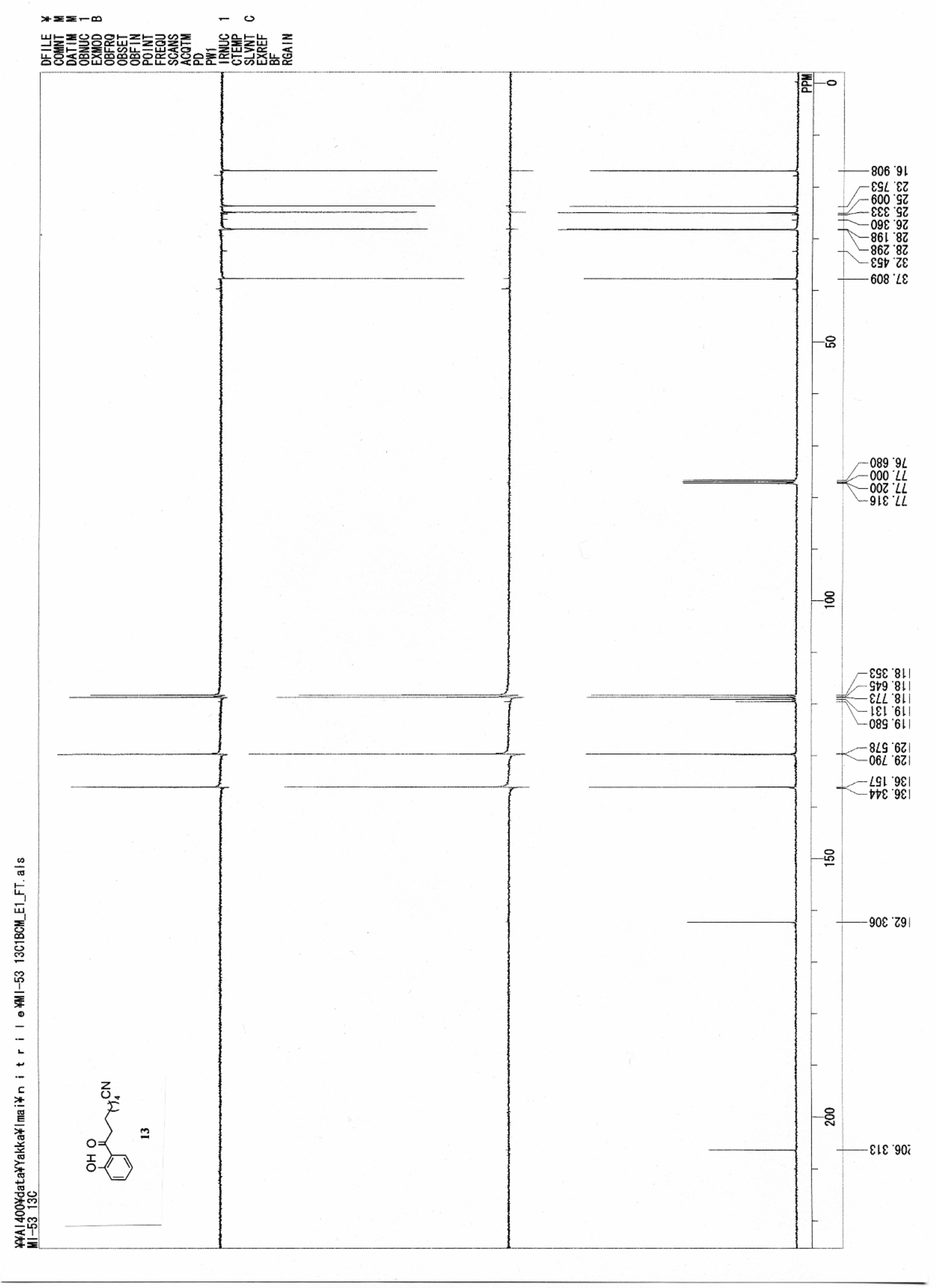




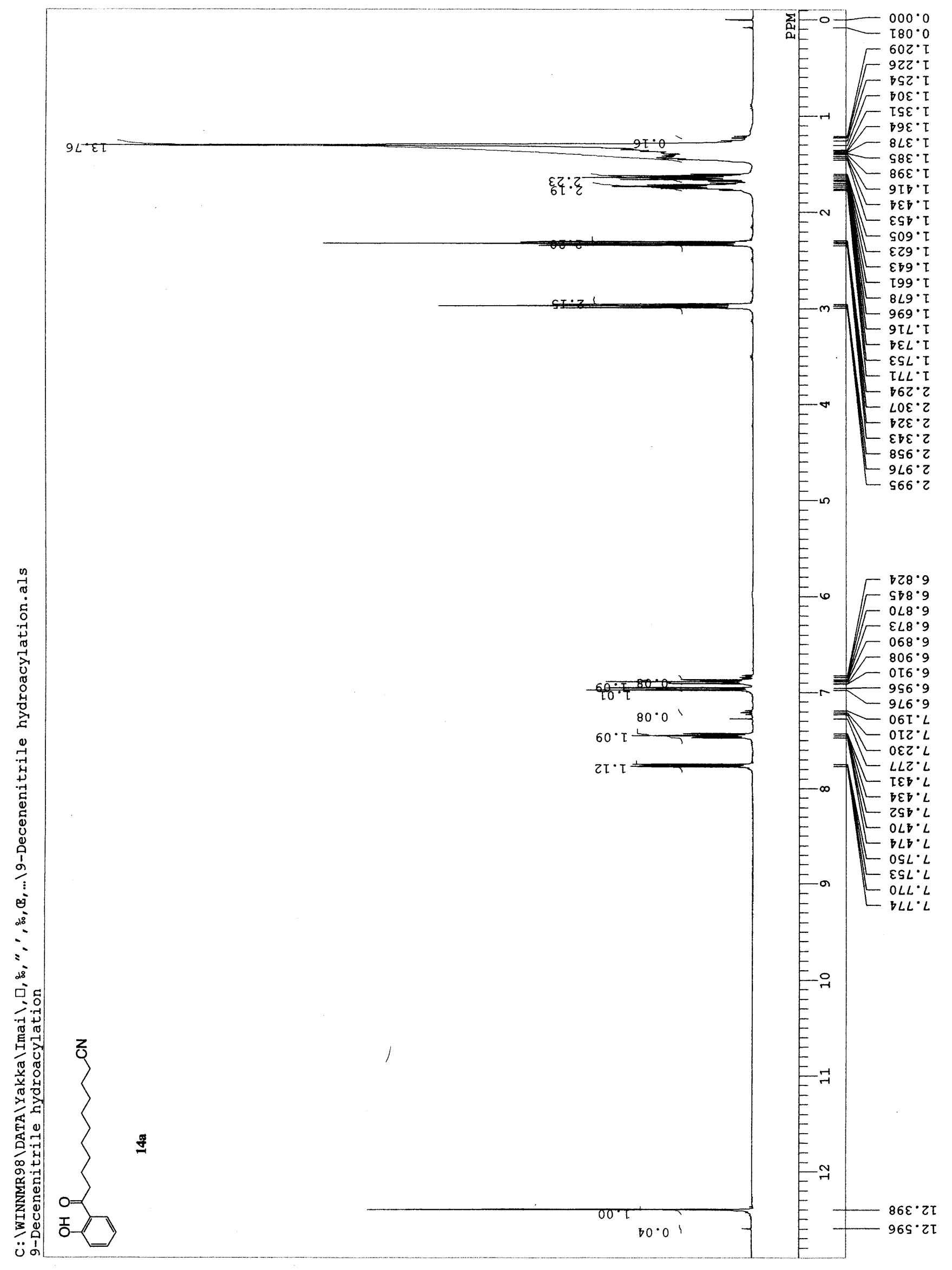




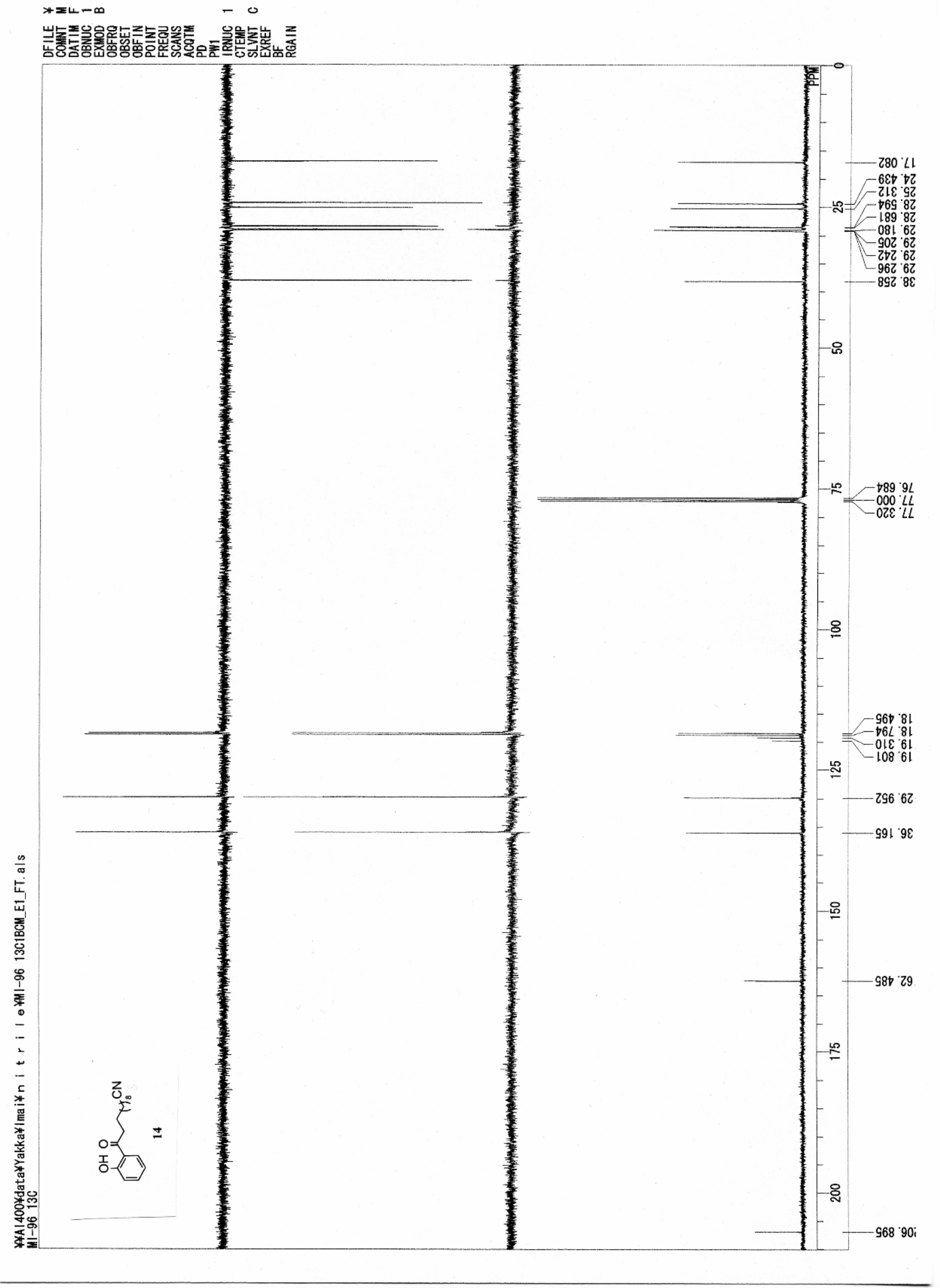

S20 


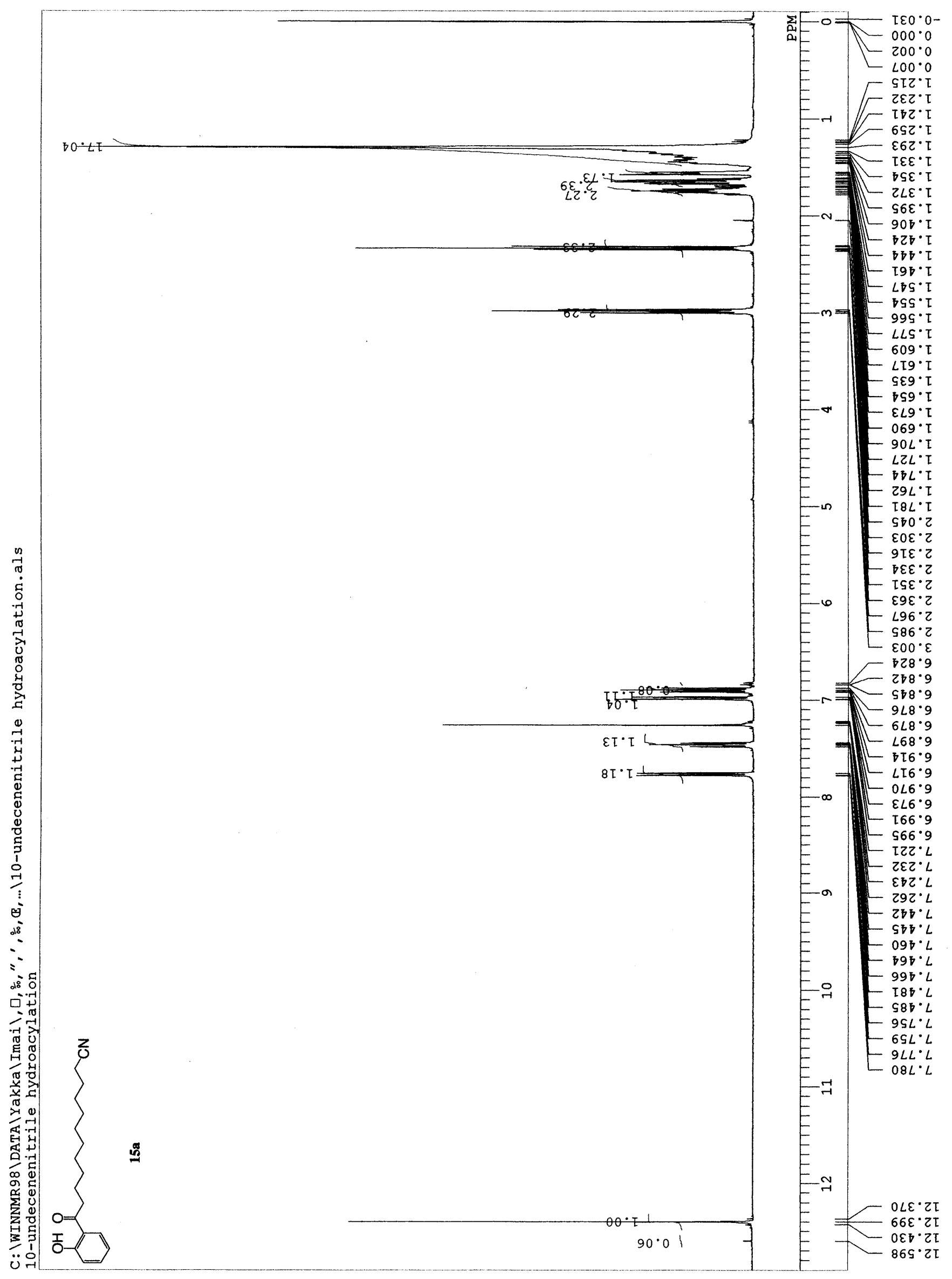




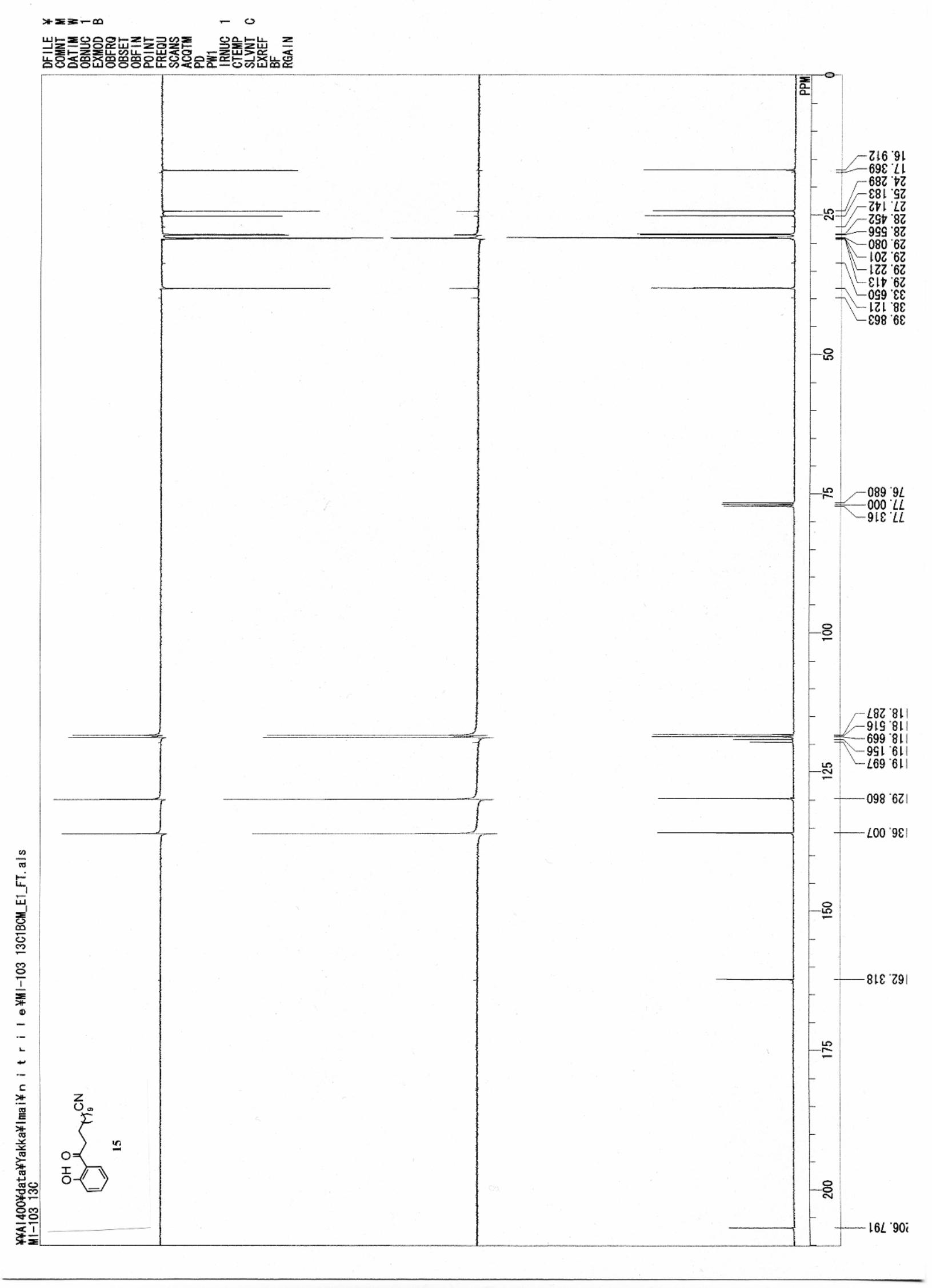




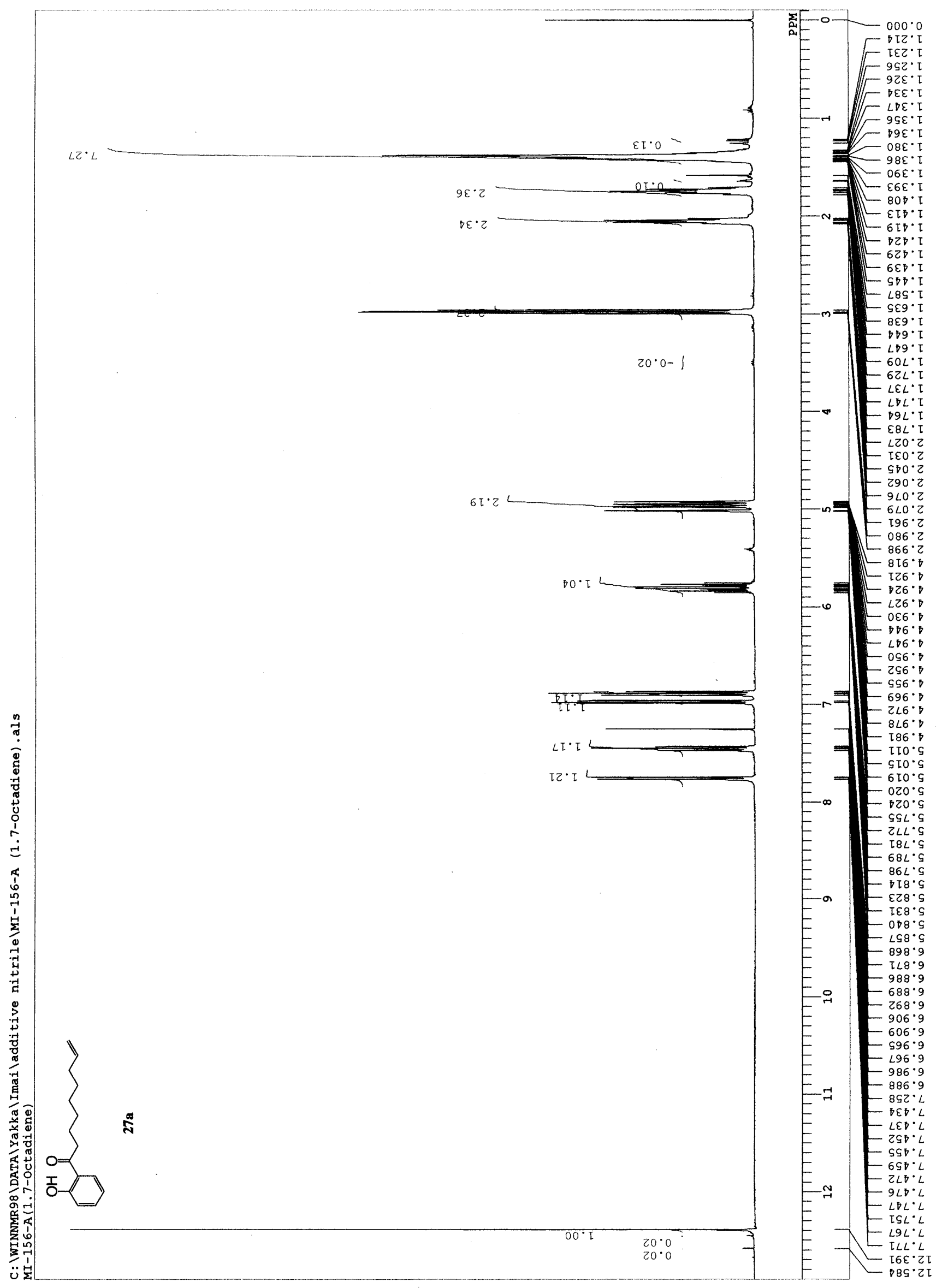




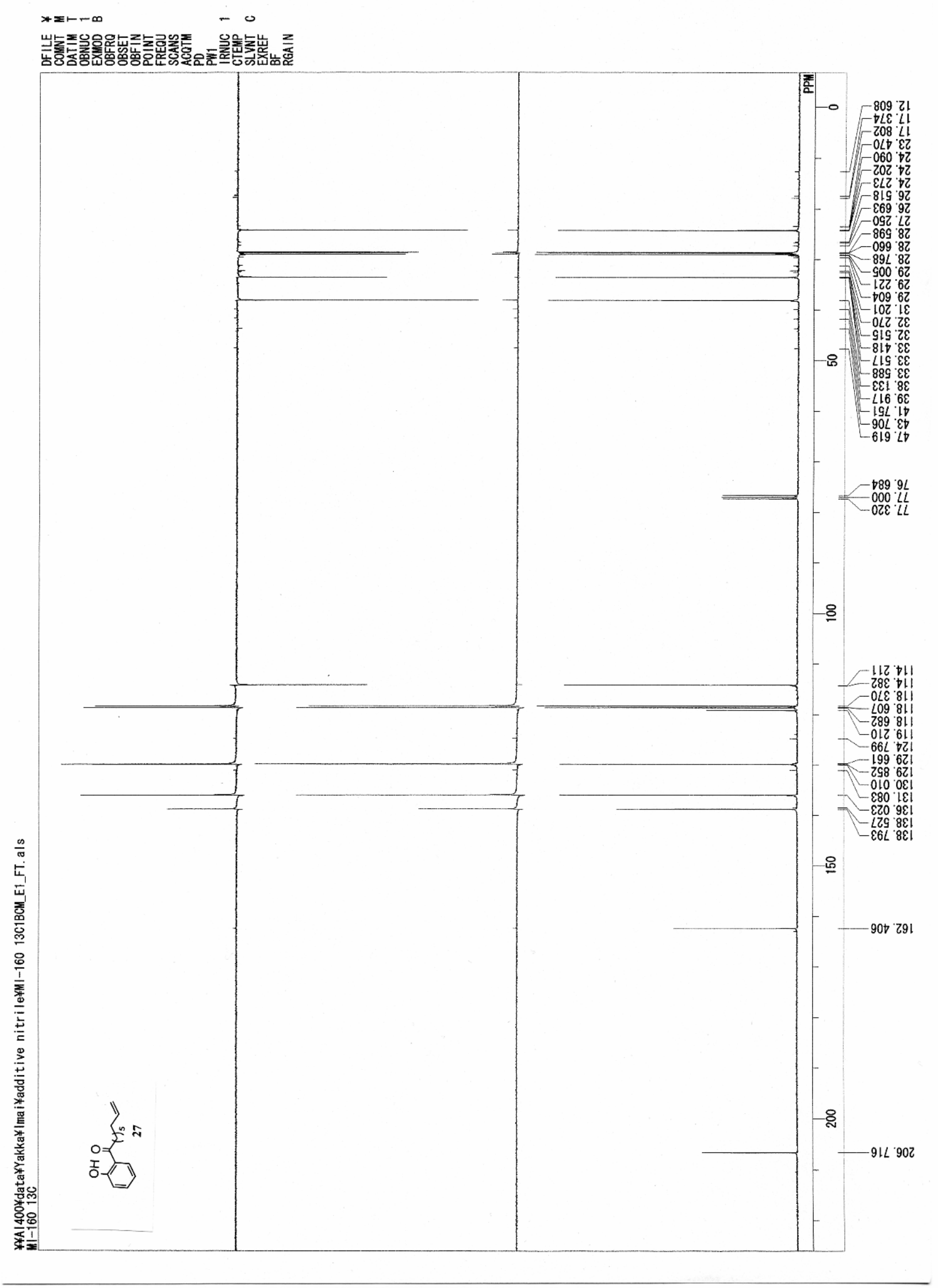




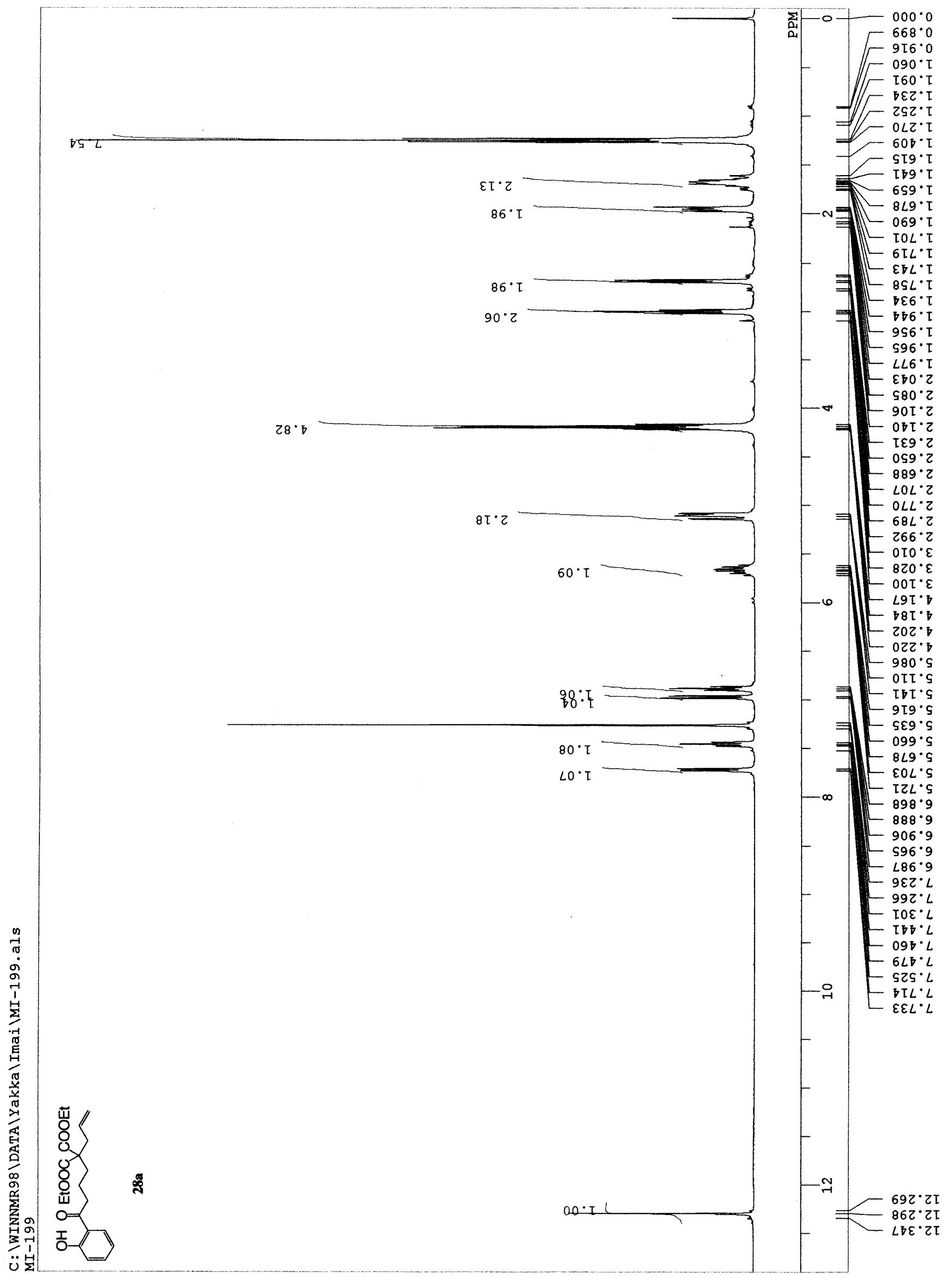




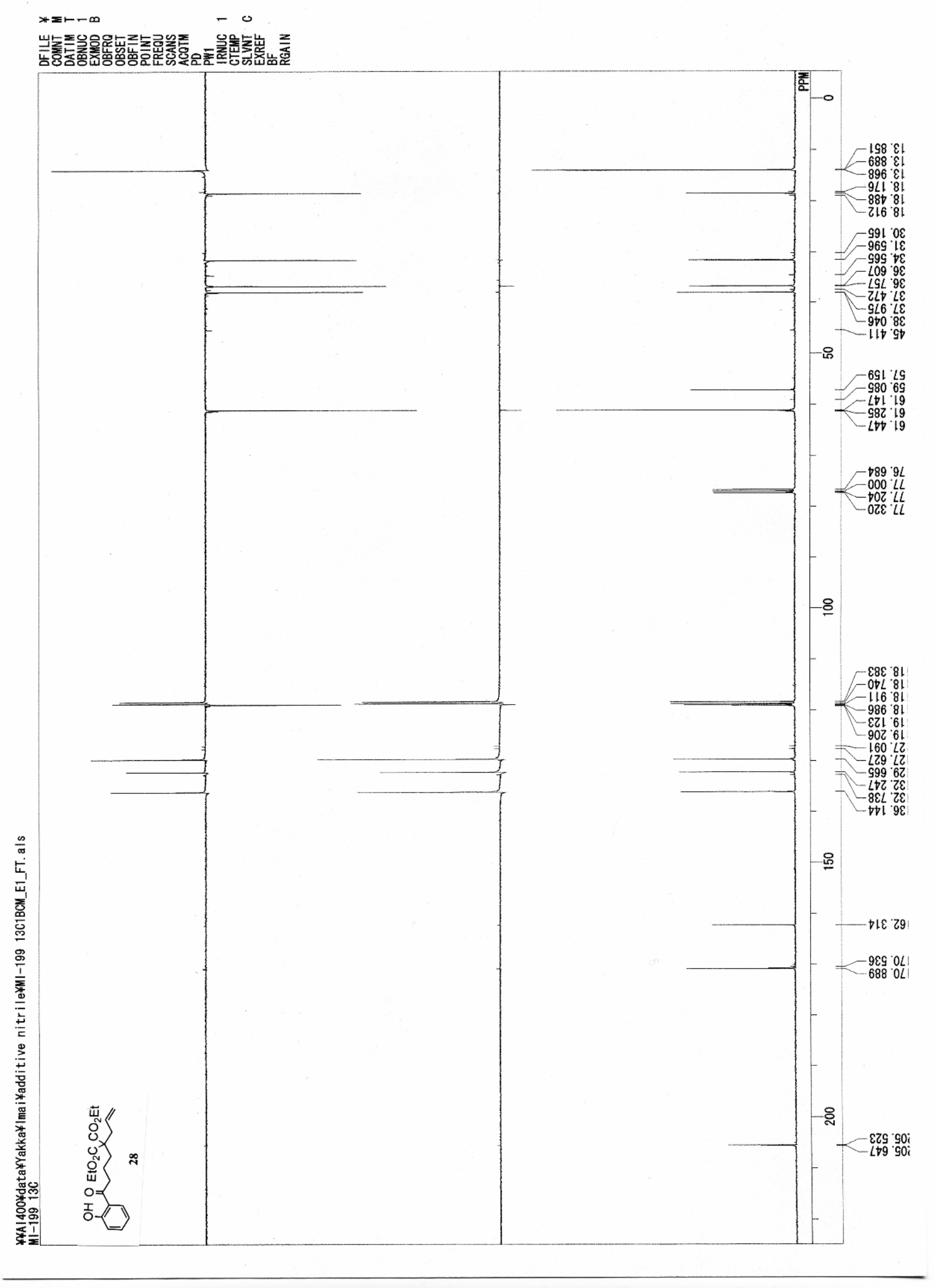




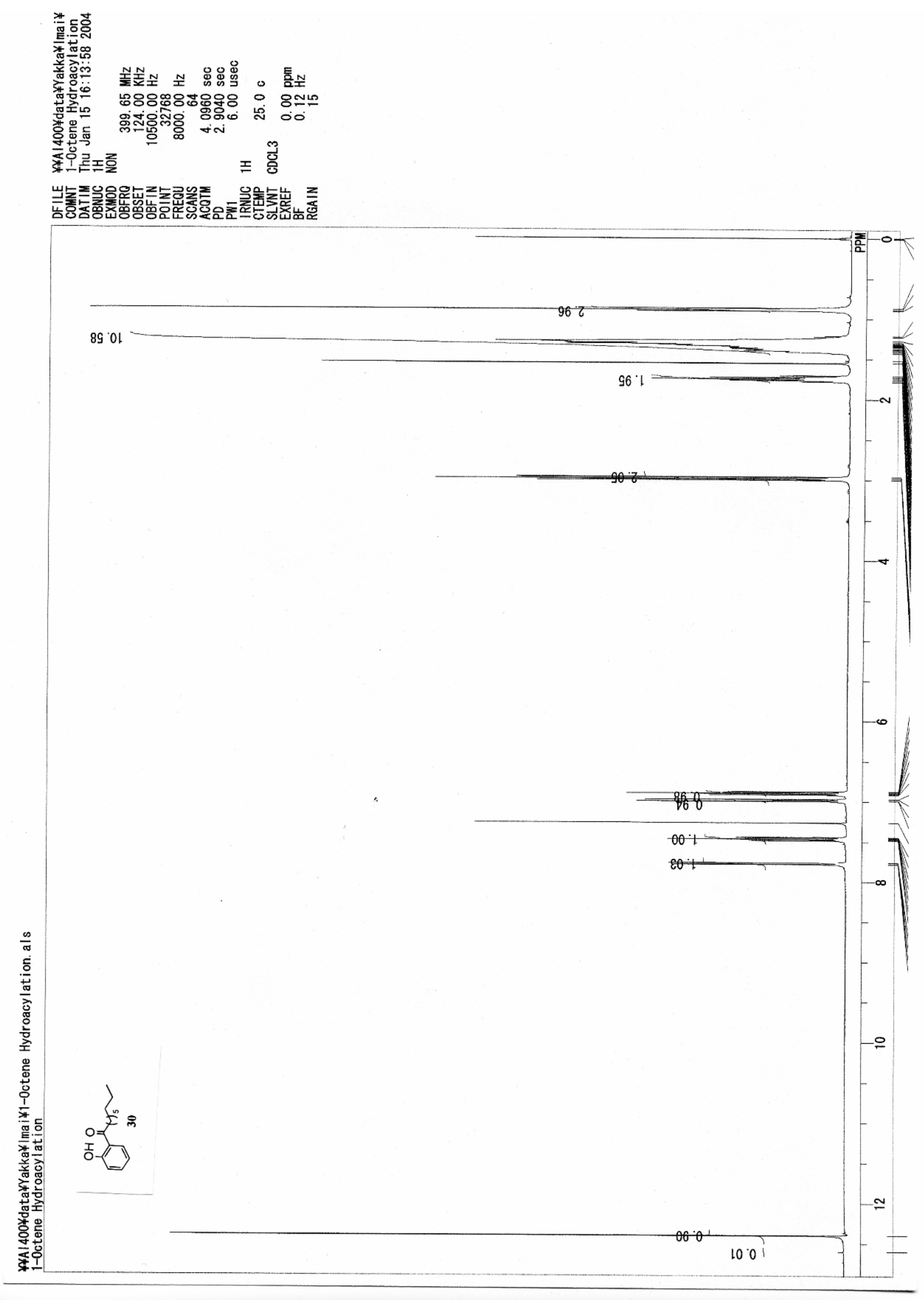




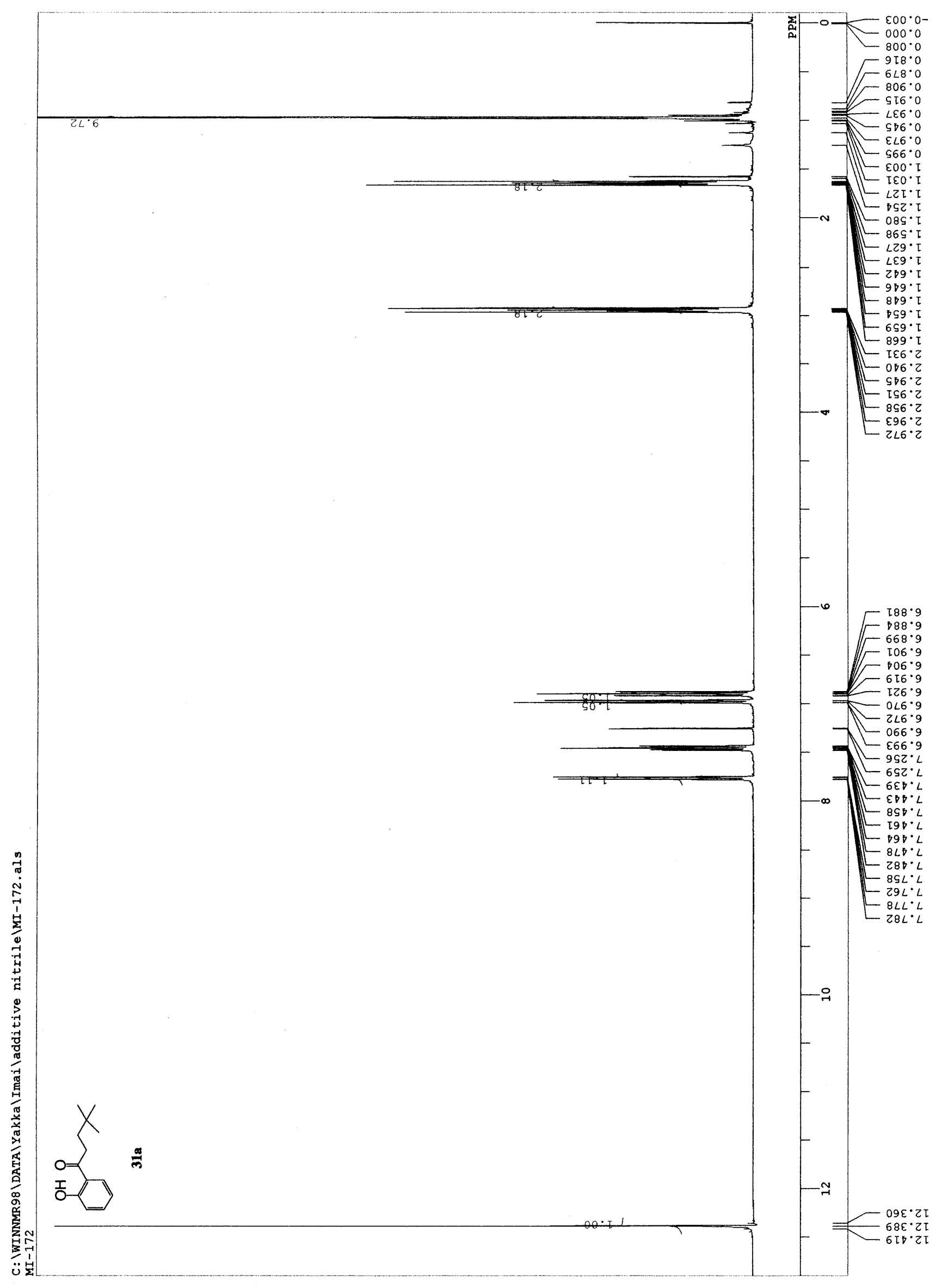




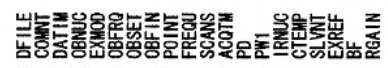

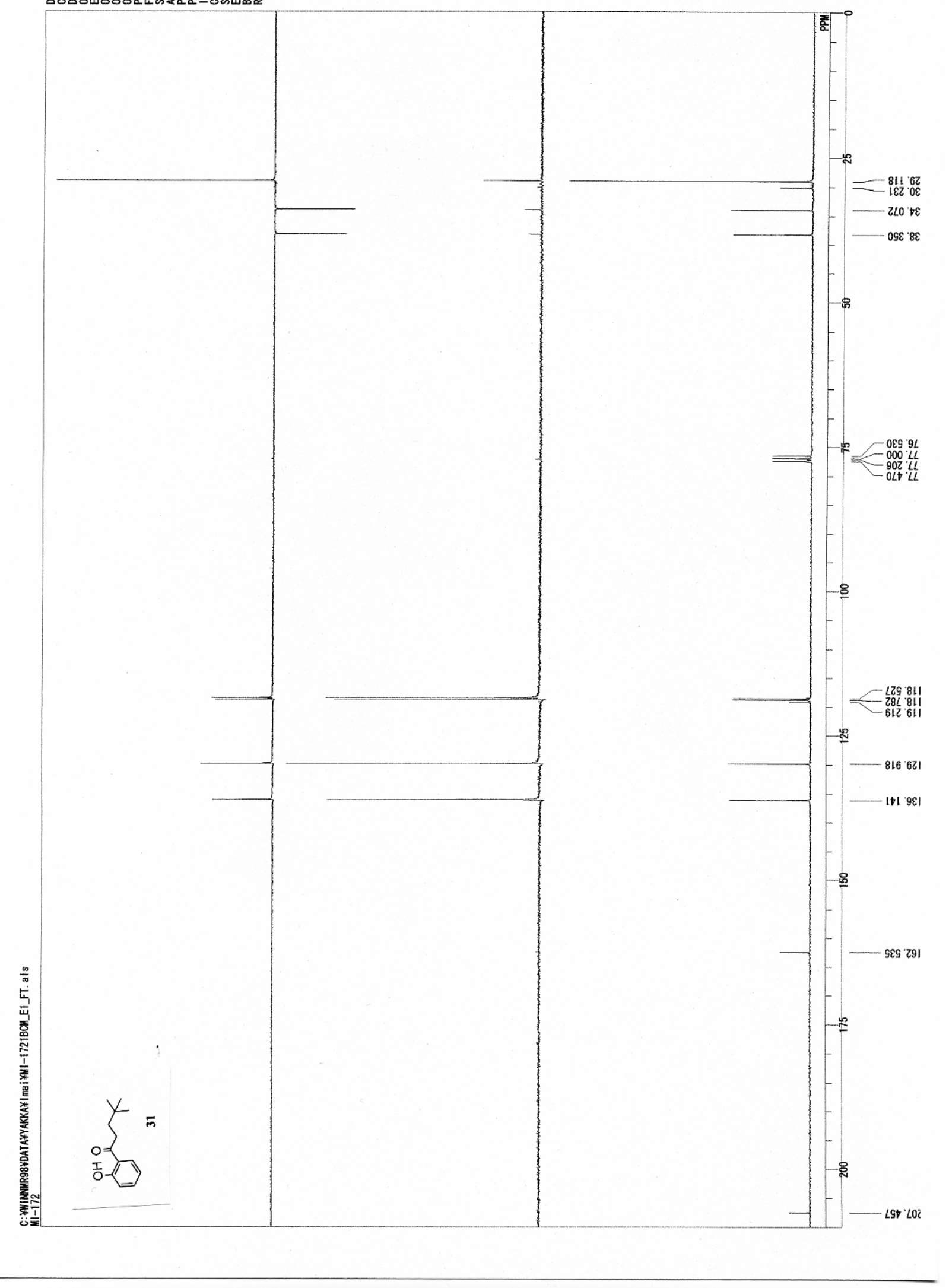




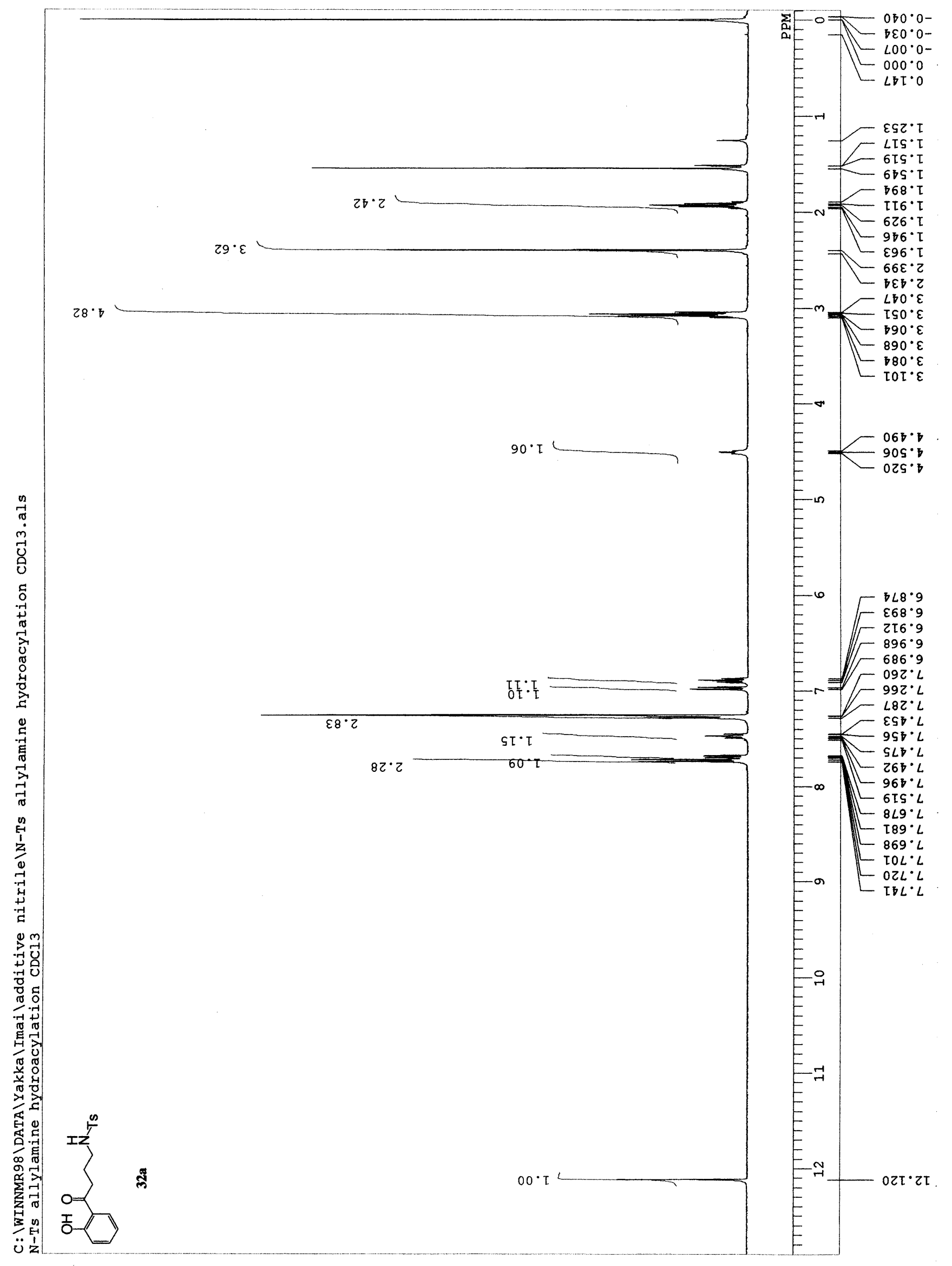




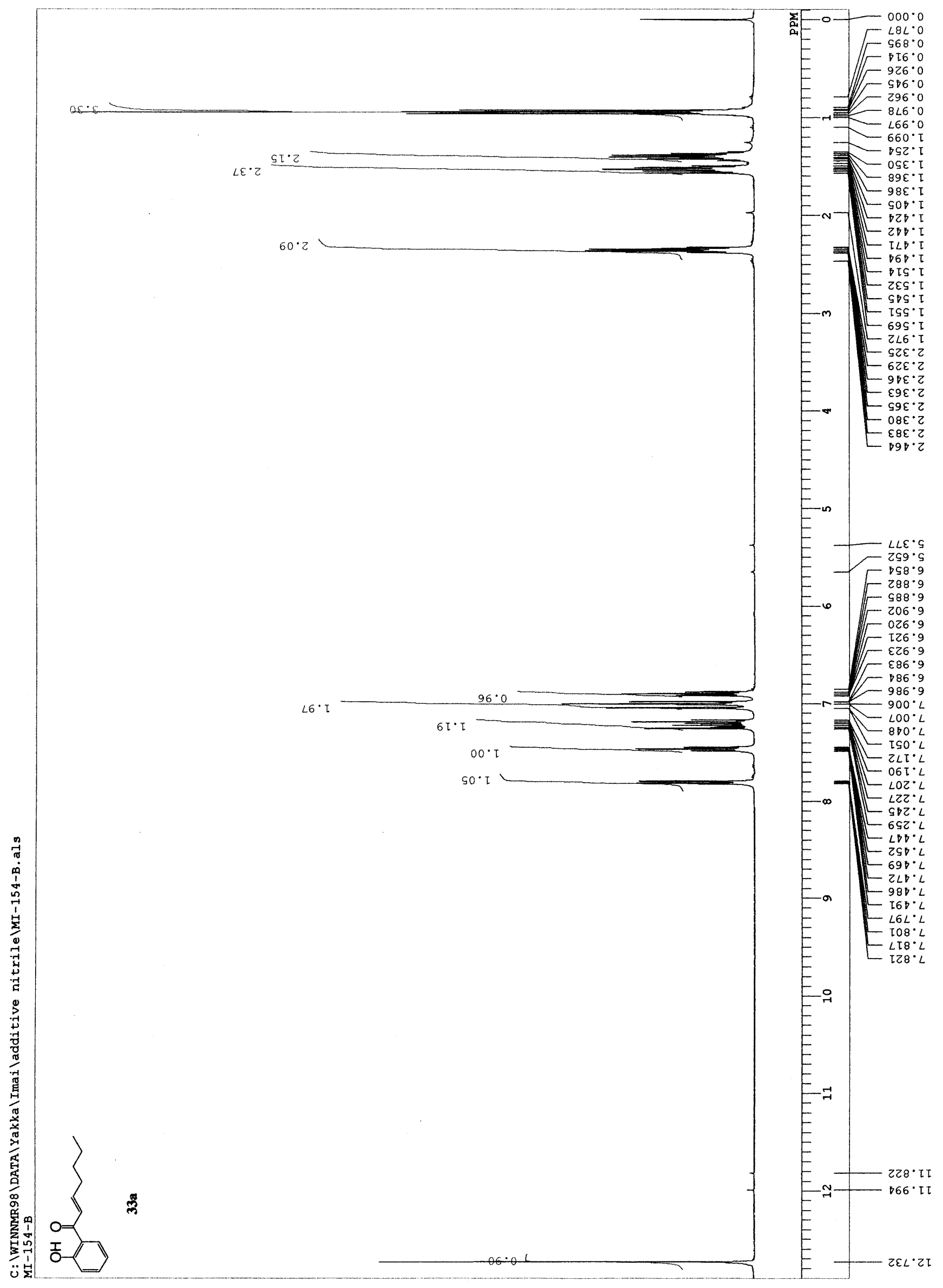




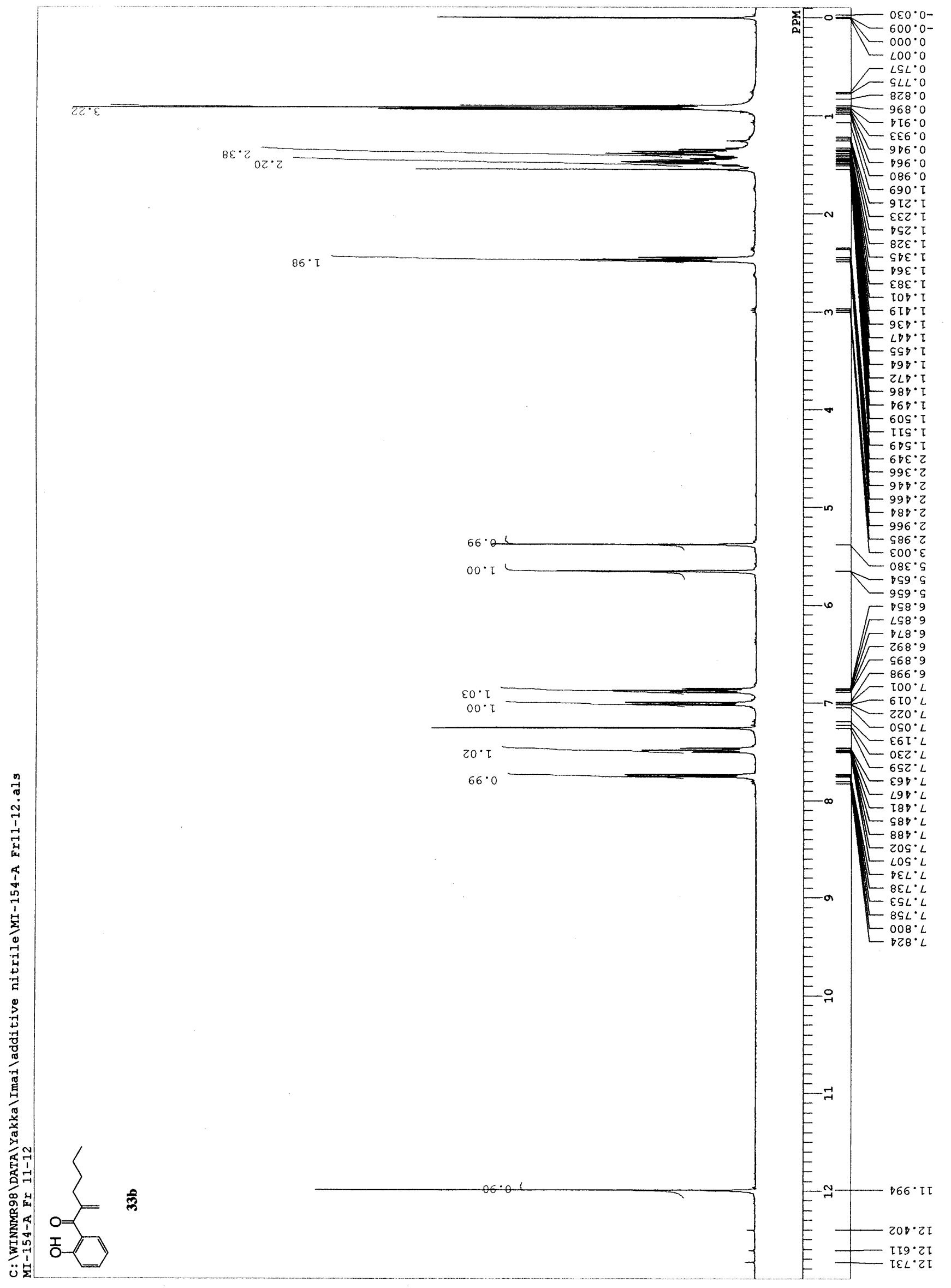

